\title{
Renormalisation of non-commutative field theories
}

\author{
Vincent Rivasseau ${ }^{a *}$ \\ Fabien Vignes-Tourneret ${ }^{b}$
}

December 18, 2008

\author{
${ }^{a}$ Laboratoire de Physique Théorique, Bât. 210 \\ Université Paris XI, F-91405 Orsay Cedex, France \\ e-mail: rivass@th.u-psud.fr \\ ${ }^{b}$ IHESS, Le Bois-Marie, 35 route de Chartres, F-91440 Bures-sur-Yvette, France \\ e-mail: vignes@ihes.fr
}

\begin{abstract}
The first renormalisable quantum field theories on non-commutative space have been found recently. We review this rapidly growing subject.
\end{abstract}

\section{Contents}

1 Introduction 2

1.1 The Quantum Hall effect . . . . . . . . . . . . . . . . . . . . . . 4

1.2 String Theory in background field . . . . . . . . . . . . . 5

2 Non-commutative field theory $\quad 6$

2.1 Field theory on Moyal space . . . . . . . . . . . . . . . . . . 6

2.1 .1 The Moyal space $\mathbb{R}_{\theta}^{D} \ldots \ldots \ldots$. . . . . . . . . . 6

2.1.2 The $\phi^{4}$-theory on $\mathbb{R}_{\theta}^{4} \ldots \ldots \ldots \ldots$. . . . . . . . . . 9

$2.1 .3 \mathrm{UV} / \mathrm{IR}$ mixing . . . . . . . . . . . . . . . . . . . . . . . 9

2.2 The Grosse-Wulkenhaar breakthrough . . . . . . . . . . . . . . . . 10

2.3 The non-commutative Gross-Neveu model . . . . . . . . . . . . . . 12

3 Multi-scale analysis in the matrix basis $\quad 14$

3.1 A dynamical matrix model . . . . . . . . . . . . . . . . . . . . . . . . 14

3.1.1 From the direct space to the matrix basis . . . . . . . . . . . . . . 14

3.1 .2 Topology of ribbon graphs . . . . . . . . . . . . . . . . . . 15

3.2 Multi-scale analysis . . . . . . . . . . . . . . . . 15

3.2.1 Bounds on the propagator . . . . . . . . . . . . 16

3.2 .2 Power counting . . . . . . . . . . . . . . . . . 17

*This review follows lectures given by V.R. at the workshop "Renormalisation et théories de Galois", Luminy, March 2006. 
3.3 Propagators on non-commutative space . . . . . . . . . . . . . . . . 19

3.3 .1 Bosonic kernel . . . . . . . . . . . . . . . . . . . . . . . . . . . . . 19

3.3 .2 Fermionic kernel . . . . . . . . . . . . . . . . . . . . 20

3.3 .3 Bounds . . . . . . . . . . . . . . . . . . . 22

3.4 Propagators and renormalisability $\ldots \ldots \ldots \ldots \ldots \ldots \ldots \ldots \ldots$

4 Direct space 23

4.1 Short and long variables . . . . . . . . . . . . . . . . . 24

4.2 Routing, Filk moves _ . . . . . . . . . . . . . . 25

4.2 .1 Oriented graphs . . . . . . . . . . . . . . . . 25

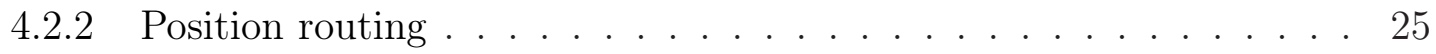

4.2 .3 Filk moves and rosettes . . . . . . . . . . . . . . . . 26

4.2 .4 Rosette factor . . . . . . . . . . . . . . . . . . . 27

4.3 Renormalisation . . . . . . . . . . . . . . . . . . . 28

4.3 .1 Four-point function . . . . . . . . . . . . . . . . . 28

4.3 .2 Two-point function . . . . . . . . . . . . . . . . . . . . . . 29

4.3.3 The Langmann-Szabo-Zarembo model . . . . . . . . . . . . . . 30

4.3.4 Critical models . . . . . . . . . . . . . . . . . . . . 30

4.4 Non-commutative hyperbolic polynomials . . . . . . . . . . . . . 32

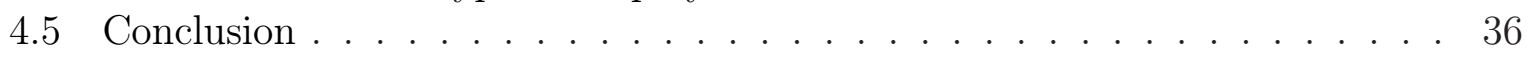

\section{Introduction}

General relativity and ordinary differential geometry should be replaced by non-commutative geometry at some point between the currently accessible energies of about 1 - $10 \mathrm{Tev}$ (after starting the Large Hadron Collider (LHC) at CERN) and the Planck scale, which is $10^{15}$ times higher, where space-time and gravity should be quantized.

This could occur either at the Planck scale or below. Quantum field theory on a noncommutative space-time (NCQF) could very well be an intermediate theory relevant for physics at energies between the LHC and the Planck scale. It certainly looks intermediate in structure between ordinary quantum field theory on commutative $\mathbb{R}^{4}$ and string theory, the current leading candidate for a more fundamental theory including quantized gravity. NCQFT in fact appears as an effective model for certain limits of string theory $[1,2]$.

In joint work with R. Gurau, J. Magnen and F. Vignes-Tourneret [3], using direct space methods, we provided recently a new proof that the Grosse-Wulkenhaar scalar $\Phi_{4}^{4}$ theory on the Moyal space $\mathbb{R}^{4}$ is renormalisable to all orders in perturbation theory.

The Grosse-Wulkenhaar breakthrough $[4,5]$ was to realize that the right propagator in non-commutative field theory is not the ordinary commutative propagator, but has to be modified to obey Langmann-Szabo duality $[6,5]$. Grosse and Wulkenhaar were able to compute the corresponding propagator in the so called "matrix base" which transforms the Moyal product into a matrix product. This is a real tour de force! They use this representation to prove perturbative renormalisability of the theory up to some estimates which were finally proven in [7].

Our direct space method builds upon the previous works of Filk and Chepelev-Roiban $[8,9]$. These works however remained inconclusive [10], since these authors used the right 
interaction but not the right propagator, hence the problem of ultraviolet/infrared mixing prevented them from obtaining a finite renormalised perturbation series.

We also extend the Grosse-Wulkenhaar results to more general models with covariant derivatives in a fixed magnetic field [11]. Our proof relies on a multiscale analysis analogous to [7] but in direct space.

Non-commutative field theories (for a general review see [12]) deserve a thorough and systematic investigation, not only because they may be relevant for physics beyond the standard model, but also (although this is often less emphasized) because they can describe effective physics in our ordinary standard world but with non-local interactions.

In this case there is an interesting reversal of the initial Grosse-Wulkenhaar problematic. In the $\Phi_{4}^{4}$ theory on the Moyal space $\mathbb{R}^{4}$, the vertex is sort of God-given by the Moyal structure, and it is LS invariant. The challenge was to overcome uv/ir mixing and to find the right propagator which makes the theory renormalisable. This propagator turned out to have LS duality. The harmonic potential introduced by Grosse and Wulkenhaar can be interpreted as a piece of covariant derivatives in a constant magnetic field.

Now to explain the (fractional) quantum Hall effect, which is a bulk effect whose understanding requires electron interactions, we can almost invert this logic. The propagator is known since it corresponds to non-relativistic electrons in two dimensions in a constant magnetic field. It has LS duality. But the interaction is unclear, and cannot be local since at strong magnetic field the spins should align with the magnetic field, hence by Pauli principle local interactions among electrons in the first Landau level should vanish.

We can argue that among all possible non-local interactions, a few renormalisation group steps should select the only ones which form a renormalisable theory with the corresponding propagator. In the commutative case (i.e. zero magnetic field) local interactions such as those of the Hubbard model are just renormalisable in any dimension because of the extended nature of the Fermi-surface singularity. Since the non-commutative electron propagator (i.e. in non zero magnetic field) looks very similar to the Grosse-Wulkenhaar propagator (it is in fact a generalization of the Langmann-Szabo-Zarembo propagator) we can conjecture that the renormalisable interaction corresponding to this propagator should be given by a Moyal product. That's why we hope that non-commutative field theory is the correct framework for a microscopic ab initio understanding of the fractional quantum Hall effect which is currently lacking.

Even for regular commutative field theory such as non-Abelian gauge theory, the strong coupling or non-perturbative regimes may be studied fruitfully through their noncommutative (i.e. non local) counterparts. This point of view is forcefully suggested in [2], where a mapping is proposed between ordinary and non-commutative gauge fields which do not preserve the gauge groups but preserve the gauge equivalent classes. We can at least remark that the effective physics of confinement should be governed by a non-local interaction, as is the case in effective strings or bags models.

In other words we propose to base physics upon the renormalisability principle, more than any other axiom. Renormalisability means genericity; only renormalisable interactions survive a few RG steps, hence only them should be used to describe generic effective physics of any kind. The search for renormalisabilty could be the powerful principle on which to orient ourselves in the jungle of all possible non-local interactions.

Renormalisability has also attracted considerable interest in the recent years as a pure mathematical structure. The work of Kreimer and Connes [13, 14, 15] recasts the 
recursive BPHZ forest formula of perturbative renormalisation in a nice Hopf algebra structure. The renormalisation group ambiguity reminds mathematicians of the Galois group ambiguity for roots of algebraic equations. Finding new renormalisable theories may therefore be important for the future of pure mathematics as well as for physics. That was forcefully argued during the Luminy workshop "Renormalisation and Galois Theory". Main open conjectures in pure mathematics such as the Riemann hypothesis $[16,17]$ or the Jacobian conjecture [18] may benefit from the quantum field theory and renormalisation group approach.

Considering that most of the Connes-Kreimer works uses dimensional regularization and the minimal dimensional renormalisation scheme, it is interesting to develop the parametric representation which generalize Schwinger's parametric representation of Feynman amplitudes to the non commutative context. It involves hyperbolic generalizations of the ordinary topological polynomials, which mathematicians call Kirchoff polynomials, and physicist call Symanzik polynomials in the quantum field theory context [19]. We plan also to work out the corresponding regularization and minimal dimensional renormalisation scheme and to recast it in a Hopf algebra structure. The corresponding structures seem richer than in ordinary field theory since they involve ribbon graphs and invariants which contain information about the genus of the surface on which these graphs live.

A critical goal to enlarge the class of renormalisable non-commutative field theories and to attack the Quantum Hall effect problem is to extend the results of Grosse-Wulkenhaar to Fermionic theories. The simplest theory, the two-dimensional Gross-Neveu model can be shown renormalisable to all orders in their Langmann-Szabo covariant versions, using either the matrix basis [20] or the direct space version developed here [21]. However the $x$-space version seems the most promising for a complete non-perturbative construction, using Pauli's principle to controll the apparent (fake) divergences of perturbation theory.

In the case of $\phi_{4}^{4}$, recall that although the commutative version is until now fatally flawed due to the famous Landau ghost, there is hope that the non-commutative field theory treated at the perturbative level in this paper may also exist at the constructive level. Indeed a non trivial fixed point of the renormalization group develops at high energy, where the Grosse-Wulkenhaar parameter $\Omega$ tends to 1, so that Langmann-Szabo duality become exact, and the beta function vanishes. This scenario has been checked explicitly to all orders of perturbation theory [22, 23, 24]. This was done using the matrix version of the theory; again an $x$-space version of renormalisation might be better for a future rigorous non-perturbative investigation of this fixed point and a full constructive version of the model.

Finally let us conclude this short introduction by reminding that a very important and difficult goal is to also extend the Grosse-Wulkenhaar breakthrough to gauge theories.

\subsection{The Quantum Hall effect}

One considers free electrons: $H_{0}=\frac{1}{2 m}(\boldsymbol{p}+e \boldsymbol{A})^{2}=\frac{\pi^{2}}{2 m}$ where $\boldsymbol{p}=m \dot{r}-e A$ is the canonical conjugate of $r$.

The moment and position $p$ and $r$ have commutators

$$
\left[p_{i}, p_{j}\right]=0, \quad\left[r_{i}, r_{j}\right]=0, \quad\left[p_{i}, r_{j}\right]=\imath \hbar \delta_{i j} .
$$


The moments $\boldsymbol{\pi}=m \dot{r}=p+e A$ have commutators

$$
\left[\pi_{i}, \pi_{j}\right]=-\imath \hbar \epsilon_{i j} e B, \quad\left[r_{i}, r_{j}\right]=0, \quad\left[\pi_{i}, r_{j}\right]=\imath \hbar \delta_{i j}
$$

One can also introduce coordinates $R_{x}, R_{y}$ corresponding to the centers of the classical trajectories

$$
R_{x}=x-\frac{1}{e B} \pi_{y}, \quad R_{y}=y+\frac{1}{e B} \pi_{x}
$$

which do not commute:

$$
\left[R_{i}, R_{j}\right]=\imath \hbar \epsilon_{i j} \frac{1}{e B}, \quad\left[\pi_{i}, R_{j}\right]=0
$$

This means that there exist Heisenberg-like relations between quantum positions.

\subsection{String Theory in background field}

One considers the string action in a generalized background

$$
\begin{aligned}
S & =\frac{1}{4 \pi \alpha^{\prime}} \int_{\Sigma}\left(g_{\mu \nu} \partial_{a} X^{\mu} \partial^{a} X^{\nu}-2 \pi i \alpha^{\prime} B_{\mu \nu} \epsilon^{a b} \partial_{a} X^{\mu} \partial_{b} X^{\nu}\right) \\
& =\frac{1}{4 \pi \alpha^{\prime}} \int_{\Sigma} g_{\mu \nu} \partial_{a} X^{\mu} \partial^{a} X^{\nu}-\frac{i}{2} \int_{\partial \Sigma} B_{\mu \nu} X^{\mu} \partial_{t} X^{\nu},
\end{aligned}
$$

where $\Sigma$ is the string worldsheet, $\partial_{t}$ is a tangential derivative along the worldsheet boundary $\partial \Sigma$ and $B_{\mu \nu}$ is an antisymmetric background tensor. The equations of motion determine the boundary conditions:

$$
g_{\mu \nu} \partial_{n} X^{\mu}+\left.2 \pi i \alpha^{\prime} B_{\mu \nu} \partial_{t} X^{\mu}\right|_{\partial \Sigma}=0 .
$$

Boundary conditions for coordinates can be Neumann $(B \rightarrow 0)$ or Dirichlet $(g \rightarrow 0$, corresponding to branes).

After conformal mapping of the string worldsheet onto the upper half-plane, the string propagator in background field is

$$
\begin{aligned}
<X^{\mu}(z) X^{\nu}\left(z^{\prime}\right)>= & -\alpha^{\prime}\left[g^{\mu \nu}\left(\log \left|z-z^{\prime}\right| \log \left|z-\bar{z}^{\prime}\right|\right)\right. \\
& \left.+G^{\mu \nu} \log \left|z-\bar{z}^{\prime}\right|^{2}+\theta^{\mu \nu} \log \frac{\left|z-\bar{z}^{\prime}\right|}{\left|\bar{z}-z^{\prime}\right|}+\text { const }\right] .
\end{aligned}
$$

for some constant symmetric and antisymmetric tensors $G$ and $\theta$.

Evaluated at boundary points on the worldsheet, this propagator is

$$
<X^{\mu}(\tau) X^{\nu}\left(\tau^{\prime}\right)>=-\alpha^{\prime} G^{\mu \nu} \log \left(\tau-\tau^{\prime}\right)^{2}+\frac{i}{2} \theta^{\mu \nu} \epsilon\left(\tau-\tau^{\prime}\right)
$$

where the $\theta$ term simply comes from the discontinuity of the logarithm across its cut. Interpreting $\tau$ as time, one finds

$$
\left[X^{\mu}, X^{\nu}\right]=i \theta^{\mu \nu}
$$

which means that string coordinates lie in a non-commutative Moyal space with parameter $\theta$.

There is an equivalent argument inspired by $M$ theory: a rotation sandwiched between two $T$ dualities generates the same constant commutator for string coordinates. 


\section{Non-commutative field theory}

\subsection{Field theory on Moyal space}

The recent progresses concerning the renormalisation of non-commutative field theory have been obtained on a very simple non-commutative space namely the Moyal space. From the point of view of quantum field theory, it is certainly the most studied space. Let us start with its precise definition.

\subsubsection{The Moyal space $\mathbb{R}_{\theta}^{D}$}

Let us define $E=\left\{x^{\mu}, \mu \in \llbracket 1, D \rrbracket\right\}$ and $\mathbb{C}\langle E\rangle$ the free algebra generated by $E$. Let $\Theta$ a $D \times D$ non-degenerate skew-symmetric matrix (wich requires $D$ even) and $I$ the ideal of $\mathbb{C}\langle E\rangle$ generated by the elements $x^{\mu} x^{\nu}-x^{\nu} x^{\mu}-\imath \Theta^{\mu \nu}$. The Moyal algebra $\mathcal{A}_{\Theta}$ is the quotient $\mathbb{C}\langle E\rangle / I$. Each element in $\mathcal{A}_{\Theta}$ is a formal power series in the $x^{\mu}$ 's for which the relation $\left[x^{\mu}, x^{\nu}\right]=\imath \Theta^{\mu \nu}$ holds.

Usually, one puts the matrix $\Theta$ into its canonical form :

$$
\Theta=\left(\begin{array}{ccccc}
0 & \theta_{1} & & (0) \\
-\theta_{1} & 0 & & & \\
& & \ddots & & \\
(0) & & 0 & \theta_{D / 2} \\
(0 & & & -\theta_{D / 2} & 0
\end{array}\right) .
$$

Sometimes one even set $\theta=\theta_{1}=\cdots=\theta_{D / 2}$. The preceeding algebraic definition whereas short and precise may be too abstract to perform real computations. One then needs a more analytical definition. A representation of the algebra $\mathcal{A}_{\Theta}$ is given by some set of functions on $\mathbb{R}^{d}$ equipped with a non-commutative product: the Groenwald-Moyal product. What follows is based on [25].

The Algebra $\mathcal{A}_{\Theta}$ The Moyal algebra $\mathcal{A}_{\Theta}$ is the linear space of smooth and rapidly decreasing functions $\mathcal{S}\left(\mathbb{R}^{D}\right)$ equipped with the non-commutative product defined by: $\forall f, g \in \mathcal{S}_{D} \stackrel{\text { def }}{=} \mathcal{S}\left(\mathbb{R}^{D}\right)$,

$$
\begin{aligned}
\left(f \star_{\Theta} g\right)(x) & =\int_{\mathbb{R}^{D}} \frac{d^{D} k}{(2 \pi)^{D}} d^{D} y f\left(x+\frac{1}{2} \Theta \cdot k\right) g(x+y) e^{\imath k \cdot y} \\
& =\frac{1}{\pi^{D}|\operatorname{det} \Theta|} \int_{\mathbb{R}^{D}} d^{D} y d^{D} z f(x+y) g(x+z) e^{-2 \imath y \Theta^{-1} z} .
\end{aligned}
$$

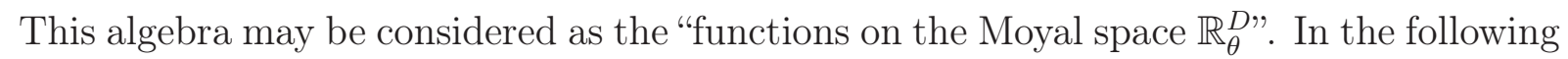
we will write $f \star g$ instead of $f \star_{\Theta} g$ and use : $\forall f, g \in \mathcal{S}_{D}, \forall j \in \llbracket 1,2 N \rrbracket$,

$$
(\mathscr{F} f)(x)=\int f(t) e^{-\imath t x} d t
$$

for the Fourier transform and

$$
(f \diamond g)(x)=\int f(x-t) g(t) e^{2 x x \Theta^{-1} t} d t
$$


for the twisted convolution. As on $\mathbb{R}^{D}$, the Fourier transform exchange product and convolution:

$$
\begin{aligned}
& \mathscr{F}(f \star g)=\mathscr{F}(f) \diamond \mathscr{F}(g) \\
& \mathscr{F}(f \diamond g)=\mathscr{F}(f) \star \mathscr{F}(g) .
\end{aligned}
$$

One also shows that the Moyal product and the twisted convolution are associative:

$$
\begin{aligned}
((f \diamond g) \diamond h)(x) & =\int f(x-t-s) g(s) h(t) e^{2 \imath\left(x \Theta^{-1} t+(x-t) \Theta^{-1} s\right)} d s d t \\
& =\int f(u-v) g(v-t) h(t) e^{2 \imath\left(x \Theta^{-1} v-t \Theta^{-1} v\right)} d t d v \\
& =(f \diamond(g \diamond h))(x) .
\end{aligned}
$$

Using (2.7), we show the associativity of the $\star$-produit. The complex conjugation is involutive in $\mathcal{A}_{\Theta}$

$$
\overline{f \star_{\Theta} g}=\bar{g} \star_{\Theta} \bar{f} .
$$

One also have

$$
f \star_{\Theta} g=g \star_{-\Theta} f
$$

Proposition 2.1 (Trace). For all $f, g \in \mathcal{S}_{D}$,

$$
\int d x(f \star g)(x)=\int d x f(x) g(x)=\int d x(g \star f)(x) .
$$

Proof.

$$
\begin{aligned}
\int d x(f \star g)(x) & =\mathscr{F}(f \star g)(0)=(\mathscr{F} f \diamond \mathscr{F} g)(0) \\
& =\int \mathscr{F} f(-t) \mathscr{F} g(t) d t=(\mathscr{F} f * \mathscr{F} g)(0)=\mathscr{F}(f g)(0) \\
& =\int f(x) g(x) d x
\end{aligned}
$$

where $*$ is the ordinary convolution.

In the following sections, we will need lemma 2.2 to compute the interaction terms for the $\Phi_{4}^{4}$ and Gross-Neveu models. We write $x \wedge y \stackrel{\text { def }}{=} 2 x \Theta^{-1} y$.

Lemma 2.2. For all $j \in \llbracket 1,2 n+1 \rrbracket$, let $f_{j} \in \mathcal{A}_{\Theta}$. Then

$$
\begin{aligned}
\left(f_{1} \star_{\Theta} \cdots \star_{\Theta} f_{2 n}\right)(x) & =\frac{1}{\pi^{2 D} \operatorname{det}^{2} \Theta} \int \prod_{j=1}^{2 n} d x_{j} f_{j}\left(x_{j}\right) e^{-\imath x \wedge \sum_{i=1}^{2 n}(-1)^{i+1} x_{i}} e^{-\imath \varphi_{2 n}} \\
\left(f_{1} \star_{\Theta} \cdots \star_{\Theta} f_{2 n+1}\right)(x) & =\frac{1}{\pi^{D} \operatorname{det} \Theta} \int \prod_{j=1}^{2 n+1} d x_{j} f_{j}\left(x_{j}\right) \delta\left(x-\sum_{i=1}^{2 n+1}(-1)^{i+1} x_{i}\right) e^{-\imath \varphi_{2 n+1}} \\
\forall p \in \mathbb{N}, \varphi_{p} & =\sum_{i<j=1}^{p}(-1)^{i+j+1} x_{i} \wedge x_{j} .
\end{aligned}
$$


Corollary 2.3. For all $j \in \llbracket 1,2 n+1 \rrbracket$, let $f_{j} \in \mathcal{A}_{\Theta}$. Then

$$
\begin{gathered}
\int d x\left(f_{1} \star_{\Theta} \cdots \star_{\Theta} f_{2 n}\right)(x)=\frac{1}{\pi^{D} \operatorname{det} \Theta} \int \prod_{j=1}^{2 n} d x_{j} f_{j}\left(x_{j}\right) \delta\left(\sum_{i=1}^{2 n}(-1)^{i+1} x_{i}\right) e^{-\imath \varphi_{2 n}}, \\
\int d x\left(f_{1} \star_{\Theta} \cdots \star_{\Theta} f_{2 n+1}\right)(x)=\frac{1}{\pi^{D} \operatorname{det} \Theta} \int \prod_{j=1}^{2 n+1} d x_{j} f_{j}\left(x_{j}\right) e^{-\imath \varphi_{2 n+1}}, \\
\forall p \in \mathbb{N}, \varphi_{p}=\sum_{i<j=1}^{p}(-1)^{i+j+1} x_{i} \wedge x_{j} .
\end{gathered}
$$

The cyclicity of the product, inherited from proposition 2.1 implies: $\forall f, g, h \in \mathcal{S}_{D}$,

$$
\langle f \star g, h\rangle=\langle f, g \star h\rangle=\langle g, h \star f\rangle
$$

and allows to extend the Moyal algebra by duality into an algebra of tempered distributions.

Extension by Duality Let us first consider the product of a tempered distribution with a Schwartz-class function. Let $T \in \mathcal{S}_{D}^{\prime}$ and $h \in \mathcal{S}_{D}$. We define $\langle T, h\rangle \stackrel{\text { def }}{=} T(h)$ and $\left\langle T^{*}, h\right\rangle=\overline{\langle T, \bar{h}\rangle}$.

Definition 2.1. Let $T \in \mathcal{S}_{D}^{\prime}, f, h \in \mathcal{S}_{D}$, we define $T \star f$ and $f \star T$ by

$$
\begin{aligned}
& \langle T \star f, h\rangle=\langle T, f \star h\rangle, \\
& \langle f \star T, h\rangle=\langle T, h \star f\rangle .
\end{aligned}
$$
$\mathcal{S}_{D}$

For example, the identity $\mathbb{1}$ as an element of $\mathcal{S}_{D}^{\prime}$ is the unity for the $\star$-produit: $\forall f, h \in$

$$
\begin{aligned}
\langle\mathbb{1} \star f, h\rangle & =\langle\mathbb{1}, f \star h\rangle \\
& =\int(f \star h)(x) d x=\int f(x) h(x) d x \\
& =\langle f, h\rangle .
\end{aligned}
$$

We are now ready to define the linear space $\mathcal{M}$ as the intersection of two sub-spaces $\mathcal{M}_{L}$ and $\mathcal{M}_{R}$ of $\mathcal{S}_{D}^{\prime}$.

Definition 2.2 (Multipliers algebra).

$$
\begin{aligned}
\mathcal{M}_{L} & =\left\{S \in \mathcal{S}_{D}^{\prime}: \forall f \in \mathcal{S}_{D}, S \star f \in \mathcal{S}_{D}\right\}, \\
\mathcal{M}_{R} & =\left\{R \in \mathcal{S}_{D}^{\prime}: \forall f \in \mathcal{S}_{D}, f \star R \in \mathcal{S}_{D}\right\}, \\
\mathcal{M} & =\mathcal{M}_{L} \cap \mathcal{M}_{R} .
\end{aligned}
$$

One can show that $\mathcal{M}$ is an associative $*$-algebra. It contains, among others, the identity, the polynomials, the $\delta$ distribution and its derivatives. Then the relation

$$
\left[x^{\mu}, x^{\nu}\right]=\imath \Theta^{\mu \nu},
$$

often given as a definition of the Moyal space, holds in $\mathcal{M}$ (but not in $\mathcal{A}_{\Theta}$ ). 


\subsubsection{The $\phi^{4}$-theory on $\mathbb{R}_{\theta}^{4}$}

The simplest non-commutative model one may consider is the $\phi^{4}$-theory on the fourdimensional Moyal space. Its Lagrangian is the usual (commutative) one where the pointwise product is replaced by the Moyal one:

$$
S[\phi]=\int d^{4} x\left(-\frac{1}{2} \partial_{\mu} \phi \star \partial^{\mu} \phi+\frac{1}{2} m^{2} \phi \star \phi+\frac{\lambda}{4} \phi \star \phi \star \phi \star \phi\right)(x) .
$$

Thanks to the formula (2.3), this action can be explicitly computed. The interaction part is given by the corollary 2.3:

$$
\begin{aligned}
\int d x \phi^{\star 4}(x) & =\int \prod_{i=1}^{4} d x_{i} \phi\left(x_{i}\right) \delta\left(x_{1}-x_{2}+x_{3}-x_{4}\right) e^{i \varphi}, \\
\varphi & =\sum_{i<j=1}^{4}(-1)^{i+j+1} x_{i} \wedge x_{j} .
\end{aligned}
$$

The main characteristic of the Moyal product is its non-locality. But its non-commutativity implies that the vertex of the model (2.28) is only invariant under cyclic permutation of the fields. This restricted invariance incites to represent the associated Feynman graphs with ribbon graphs. One can then make a clear distinction between planar and non-planar graphs. This will be detailed in section 3 .

Thanks to the delta function in (2.29), the oscillation may be written in different ways:

$$
\begin{aligned}
\delta\left(x_{1}-x_{2}+x_{3}-x_{4}\right) e^{\imath \varphi} & =\delta\left(x_{1}-x_{2}+x_{3}-x_{4}\right) e^{\imath x_{1} \wedge x_{2}+\imath x_{3} \wedge x_{4}} \\
& =\delta\left(x_{1}-x_{2}+x_{3}-x_{4}\right) e^{\imath x_{4} \wedge x_{1}+\imath x_{2} \wedge x_{3}} \\
& =\delta\left(x_{1}-x_{2}+x_{3}-x_{4}\right) \exp \imath\left(x_{1}-x_{2}\right) \wedge\left(x_{2}-x_{3}\right) .
\end{aligned}
$$

The interaction is real and positive ${ }^{1}$ :

$$
\begin{aligned}
& \int \prod_{i=1}^{4} d x_{i} \phi\left(x_{i}\right) \delta\left(x_{1}-x_{2}+x_{3}-x_{4}\right) e^{\imath \varphi} \\
= & \int d k\left(\int d x d y \phi(x) \phi(y) e^{\imath k(x-y)+\imath x \wedge y}\right)^{2} \in \mathbb{R}_{+} .
\end{aligned}
$$

It is also translation invariant as shows equation (2.30c).

The property 2.1 implies that the propagator is the usual one: $\hat{C}(p)=1 /\left(p^{2}+m^{2}\right)$.

\subsubsection{UV/IR mixing}

The non-locality of the $\star$-product allows to understand the discovery of Minwalla, Van Raamsdonk and Seiberg [26]. They showed that not only the model (2.28) isn't finite in the UV but also it exhibits a new type of divergences making it non-renormalisable. In the article [8], Filk computed the Feynman rules corresponding to (2.28). He showed that the planar amplitudes equal the commutative ones whereas the non-planar ones give

\footnotetext{
${ }^{1}$ Another way to prove it is from $(2.10), \overline{\phi^{\star 4}}=\phi^{\star 4}$.
} 
rise to oscillations coupling the internal and external legs. A typical example is the the non-planar tadpole:

$$
\begin{aligned}
\longrightarrow p\left(-\frac{\lambda}{12} \int \frac{d^{4} k}{(2 \pi)^{4}} \frac{e^{i p_{\mu} k_{\nu} \Theta^{\mu \nu}}}{k^{2}+m^{2}}\right. \\
=\frac{\lambda}{48 \pi^{2}} \sqrt{\frac{m^{2}}{(\Theta p)^{2}}} K_{1}\left(\sqrt{m^{2}(\Theta p)^{2}}\right) \underset{p \rightarrow 0}{\simeq} p^{-2} .
\end{aligned}
$$

If $p \neq 0$, this amplitude is finite but, for small $p$, it diverges like $p^{-2}$. In other words, if we put an ultraviolet cut-off $\Lambda$ to the $k$-integral, the two limits $\Lambda \rightarrow \infty$ and $p \rightarrow 0$ do not commute. This is the UV/IR mixing phenomena. A chain of non-planar tadpoles, inserted in bigger graphs, makes divergent any function (with six points or more for example). But this divergence is not local and can't be absorbed in a mass redefinition. This is what makes the model non-renormalisable. We will see in sections 3.4 and 4 that the UV/IR mixing results in a coupling of the different scales of the theory. We will also note that we should distinguish different types of mixing.

The UV/IR mixing was studied by several groups. First, Chepelev and Roiban [9] gave a power counting for different scalr models. They were able to identify the divergent graphs and to classify the divergences of the theories thanks to the topological data of the graphs. Then V. Gayral [27] showed that UV/IR mixing is present on all isospectral deformations (they consist in curved generalisations of the Moyal space and of the non-commutative torus). For this, he considered a scalar model (2.28) and discovered contributions to the effective action which diverge when the external momenta vanish. The UV/IR mixing is then a general characteristic of the non-commutative theories, at least on the deformations.

\subsection{The Grosse-Wulkenhaar breakthrough}

The situation remained so until H. Grosse and R. Wulkenhaar discovered a way to define a renormalisable non-commutative model. We will detail their result in section 3 but the main message is the following. By adding an harmonic term to the Lagrangian (2.28),

$$
S[\phi]=\int d^{4} x\left(-\frac{1}{2} \partial_{\mu} \phi \star \partial^{\mu} \phi+\frac{\Omega^{2}}{2}\left(\tilde{x}_{\mu} \phi\right) \star\left(\tilde{x}^{\mu} \phi\right)+\frac{1}{2} m^{2} \phi \star \phi+\frac{\lambda}{4} \phi \star \phi \star \phi \star \phi\right)(x)
$$

where $\widetilde{x}=2 \Theta^{-1} x$ and the metric is Euclidean, the model, in four dimensions, is renormalisable at all orders of perturbation [5]. We will see in section 4 that this additional term give rise to an infrared cut-off and allows to decouple the different scales of the theory. The new model (2.33), we call it $\Phi_{4}^{4}$, do not exhibit any mixing. This result is very important because it opens the way towards other non-commutative field theories. In the following, we will call vulcanisation ${ }^{2}$ the procedure consisting in adding a

\footnotetext{
${ }^{2}$ TECHNOL. Opération consistant à traiter le caoutchouc naturel ou synthétique par addition de soufre, pour en améliorer les propriétés mécaniques et la résistance aux variations de température, Trésor de la Langue Française informatisé, http://www.lexilogos.com/.
} 
new term to a Lagrangian of a non-commutative theory in order to make it renormalisable.

The propagator $C$ of this $\Phi^{4}$ theory is the kernel of the inverse operator $-\Delta+\Omega^{2} \widetilde{x}^{2}+m^{2}$. It is known as the Mehler kernel [28, 20]

$$
C(x, y)=\frac{\Omega^{2}}{\theta^{2} \pi^{2}} \int_{0}^{\infty} \frac{d t}{\sinh ^{2}(2 \widetilde{\Omega} t)} e^{-\frac{\widetilde{\Omega}}{2} \operatorname{coth}(2 \widetilde{\Omega} t)(x-y)^{2}-\frac{\widetilde{\Omega}}{2} \tanh (2 \widetilde{\Omega} t)(x+y)^{2}-m^{2} t} .
$$

Langmann and Szabo remarked that the quartic interaction with Moyal product is invariant under a duality transformation. It is a symmetry between momentum and direct space. The interaction part of the model (2.33) is (see equation (2.17))

$$
\begin{aligned}
S_{\mathrm{int}}[\phi] & =\int d^{4} x \frac{\lambda}{4}(\phi \star \phi \star \phi \star \phi)(x) \\
& =\int \prod_{a=1}^{4} d^{4} x_{a} \phi\left(x_{a}\right) V\left(x_{1}, x_{2}, x_{3}, x_{4}\right) \\
& =\int \prod_{a=1}^{4} \frac{d^{4} p_{a}}{(2 \pi)^{4}} \hat{\phi}\left(p_{a}\right) \hat{V}\left(p_{1}, p_{2}, p_{3}, p_{4}\right)
\end{aligned}
$$

with

$$
\begin{aligned}
V\left(x_{1}, x_{2}, x_{3}, x_{4}\right) & =\frac{\lambda}{4} \frac{1}{\pi^{4} \operatorname{det} \Theta} \delta\left(x_{1}-x_{2}+x_{3}-x_{4}\right) \cos \left(2\left(\Theta^{-1}\right)_{\mu \nu}\left(x_{1}^{\mu} x_{2}^{\nu}+x_{3}^{\mu} x_{4}^{\nu}\right)\right) \\
\hat{V}\left(p_{1}, p_{2}, p_{3}, p_{4}\right) & =\frac{\lambda}{4}(2 \pi)^{4} \delta\left(p_{1}-p_{2}+p_{3}-p_{4}\right) \cos \left(\frac{1}{2} \Theta^{\mu \nu}\left(p_{1, \mu} p_{2, \nu}+p_{3, \mu} p_{4, \nu}\right)\right)
\end{aligned}
$$

where we used a cyclic Fourier transform: $\hat{\phi}\left(p_{a}\right)=\int d x e^{(-1)^{a} \imath p_{a} x_{a}} \phi\left(x_{a}\right)$. The transformation

$$
\hat{\phi}(p) \leftrightarrow \pi^{2} \sqrt{|\operatorname{det} \Theta|} \phi(x), \quad p_{\mu} \leftrightarrow \widetilde{x}_{\mu}
$$

exchanges (2.36) and (2.37). In addition, the free part of the model (2.28) isn't covariant under this duality. The vulcanisation adds a term to the Lagrangian which restores the symmetry. The theory (2.33) is then covariant under the Langmann-Szabo duality:

$$
S[\phi ; m, \lambda, \Omega] \mapsto \Omega^{2} S\left[\phi ; \frac{m}{\Omega}, \frac{\lambda}{\Omega^{2}}, \frac{1}{\Omega}\right] .
$$

By symmetry, the parameter $\Omega$ is confined in $[0,1]$. Let us note that for $\Omega=1$, the model is invariant.

The interpretation of that harmonic term is not yet clear. But the vulcanisation procedure already allowed to prove the renormalisability of several other models on Moyal spaces such that $\phi_{2}^{4}[29], \phi_{2,4}^{3}[30,31]$ and the LSZ models $[11,32,33]$. These last are of the type

$$
S[\phi]=\int d^{n} x\left(\frac{1}{2} \bar{\phi} \star\left(-\partial_{\mu}+\widetilde{x}_{\mu}+m\right)^{2} \phi+\frac{\lambda}{4} \bar{\phi} \star \phi \star \bar{\phi} \star \phi\right)(x) .
$$


By comparison with (2.33), one notes that here the additional term is formally equivalent to a fixed magnetic background. Deep is the temptation to interpret it as such. This model is invariant under the above duality and is exactly soluble. Let us remark that the complex interaction in (2.40) makes the Langmann-Szabo duality more natural. It doesn't need a cyclic Fourier transform. The $\phi^{3}$ have been studied at $\Omega=1$ where they also exhibit a soluble structure.

\subsection{The non-commutative Gross-Neveu model}

Apart from the $\Phi_{4}^{4}$, the modified Bosonic LSZ model [3] and supersymmetric theories, we now know several renormalizable non-commutative field theories. Nevertheless they either are super-renormalizable $\left(\Phi_{2}^{4}[29]\right)$ or (and) studied at a special point in the parameter space where they are solvable $\left(\Phi_{2}^{3}, \Phi_{4}^{3}[30,31]\right.$, the LSZ models [11, 32, 33]). Although only logarithmically divergent for parity reasons, the non-commutative Gross-Neveu model is a just renormalizable quantum field theory as $\Phi_{4}^{4}$. One of its main interesting features is that it can be interpreted as a non-local Fermionic field theory in a constant magnetic background. Then apart from strengthening the "vulcanization" procedure to get renormalizable non-commutative field theories, the Gross-Neveu model may also be useful for the study of the quantum Hall effect. It is also a good first candidate for a constructive study [34] of a non-commutative field theory as Fermionic models are usually easier to construct. Moreover its commutative counterpart being asymptotically free and exhibiting dynamical mass generation $[35,36,37]$, a study of the physics of this model would be interesting.

The non-commutative Gross-Neveu model $\left(\mathrm{GN}_{\Theta}^{2}\right)$ is a Fermionic quartically interacting quantum field theory on the Moyal plane $\mathbb{R}_{\theta}^{2}$. The skew-symmetric matrix $\Theta$ is

$$
\Theta=\left(\begin{array}{cc}
0 & -\theta \\
\theta & 0
\end{array}\right)
$$

The action is

$$
S[\bar{\psi}, \psi]=\int d x\left(\bar{\psi}\left(-\imath \not \partial+\Omega \not{x}+m+\mu \gamma_{5}\right) \psi+V_{\mathrm{o}}(\bar{\psi}, \psi)+V_{\mathrm{no}}(\bar{\psi}, \psi)\right)(x)
$$

where $\widetilde{x}=2 \Theta^{-1} x, \gamma_{5}=\imath \gamma^{0} \gamma^{1}$ and $V=V_{\mathrm{o}}+V_{\text {no }}$ is the interaction part given hereafter. The $\mu$-term appears at two-loop order. We use a Euclidean metric and the Feynman convention $\not \alpha=\gamma^{\mu} a_{\mu}$. The $\gamma^{0}$ and $\gamma^{1}$ matrices form a two-dimensional representation of the Clifford algebra $\left\{\gamma^{\mu}, \gamma^{\nu}\right\}=-2 \delta^{\mu \nu}$. Let us remark that the $\gamma^{\mu}$ 's are then skewHermitian: $\gamma^{\mu \dagger}=-\gamma^{\mu}$.

Propagator The propagator corresponding to the action (2.42) is given by the following lemma: 
Lemma 2.4 (Propagator [20]). The propagator of the Gross-Neveu model is

$$
\begin{aligned}
C(x, y)= & \int d \mu_{C}(\bar{\psi}, \psi) \psi(x) \bar{\psi}(y)=\left(-\imath \not \partial+\Omega \not x^{-1}+m\right)^{-1}(x, y) \\
= & \int_{0}^{\infty} d t C(t ; x, y), \\
C(t ; x, y)= & -\frac{\Omega}{\theta \pi} \frac{e^{-t m^{2}}}{\sinh (2 \widetilde{\Omega} t)} e^{-\frac{\widetilde{\Omega}}{2} \operatorname{coth}(2 \widetilde{\Omega} t)(x-y)^{2}+\imath \Omega x \wedge y} \\
& \times\{\imath \widetilde{\Omega} \operatorname{coth}(2 \widetilde{\Omega} t)(\not x-\not)+\Omega(\not{t}-\widetilde{y})-m\} e^{-2 \imath \Omega t \gamma \Theta^{-1} \gamma}
\end{aligned}
$$

with $\widetilde{\Omega}=\frac{2 \Omega}{\theta}$ et $x \wedge y=2 x \Theta^{-1} y$.

We also have $e^{-2 \imath \Omega t \gamma \Theta^{-1} \gamma}=\cosh (2 \widetilde{\Omega} t) \mathbb{1}_{2}-\imath \frac{\theta}{2} \sinh (2 \widetilde{\Omega} t) \gamma \Theta^{-1} \gamma$.

If we want to study a $N$-color model, we can consider a propagator diagonal in these color indices.

Interactions Concerning the interaction part $V$, recall that (see corollary 2.3) for any $f_{1}, f_{2}, f_{3}, f_{4}$ in $\mathcal{A}_{\Theta}$,

$$
\begin{aligned}
\int d x\left(f_{1} \star f_{2} \star f_{3} \star f_{4}\right)(x) & =\frac{1}{\pi^{2} \operatorname{det} \Theta} \int \prod_{j=1}^{4} d x_{j} f_{j}\left(x_{j}\right) \delta\left(x_{1}-x_{2}+x_{3}-x_{4}\right) e^{-\imath \varphi}, \\
\varphi & =\sum_{i<j=1}^{4}(-1)^{i+j+1} x_{i} \wedge x_{j} .
\end{aligned}
$$

This product is non-local and only invraiant under cyclic permutations of the fields. Then, contrary to the commutative Gross-Neveu model, for which there exits only one spinorial interaction, the $\mathrm{GN}_{\Theta}^{2}$ model has, at least, six different interacitons: the orientable ones

$$
\begin{aligned}
V_{\mathrm{o}}= & \frac{\lambda_{1}}{4} \int d x(\bar{\psi} \star \psi \star \bar{\psi} \star \psi)(x) \\
& +\frac{\lambda_{2}}{4} \int d x\left(\bar{\psi} \star \gamma^{\mu} \psi \star \bar{\psi} \star \gamma_{\mu} \psi\right)(x) \\
& +\frac{\lambda_{3}}{4} \int d x\left(\bar{\psi} \star \gamma_{5} \psi \star \bar{\psi} \star \gamma_{5} \psi\right)(x),
\end{aligned}
$$

where $\psi$ 's and $\bar{\psi}$ 's alternate and the non-orientable ones

$$
\begin{aligned}
V_{\text {no }}= & \frac{\lambda_{4}}{4} \int d x(\psi \star \bar{\psi} \star \bar{\psi} \star \psi)(x) \\
& +\frac{\lambda_{5}}{4} \int d x\left(\psi \star \gamma^{\mu} \bar{\psi} \star \bar{\psi} \star \gamma_{\mu} \psi\right)(x) \\
& +\frac{\lambda_{6}}{4} \int d x\left(\psi \star \gamma_{5} \bar{\psi} \star \bar{\psi} \star \gamma_{5} \psi\right)(x) .
\end{aligned}
$$

All these interactions have the same $x$ kernel thanks to the equation (2.45). The reason for which we call these interactions orientable or not will be clear in section 4 . 


\section{Multi-scale analysis in the matrix basis}

The matrix basis is a basis for Schwartz-class functions. In this basis, the Moyal product becomes a simple matrix product. Each field is then represented by an infinite matrix $[25,29,38]$.

\subsection{A dynamical matrix model}

\subsubsection{From the direct space to the matrix basis}

In the matrix basis, the action (2.33) takes the form:

$$
S[\phi]=(2 \pi)^{D / 2} \sqrt{\operatorname{det} \Theta}\left(\frac{1}{2} \phi \Delta \phi+\frac{\lambda}{4} \operatorname{Tr} \phi^{4}\right)
$$

where $\phi=\phi_{m n}, m, n \in \mathbb{N}^{D / 2}$ and

$$
\begin{aligned}
& \Delta_{m n, k l}=\sum_{i=1}^{D / 2}\left(\mu_{0}^{2}+\frac{2}{\theta}\left(m_{i}+n_{i}+1\right)\right) \delta_{m l} \delta_{n k} \\
& -\frac{2}{\theta}\left(1-\Omega^{2}\right)\left(\sqrt{\left(m_{i}+1\right)\left(n_{i}+1\right)} \delta_{m_{i}+1, l_{i}} \delta_{n_{i}+1, k_{i}}+\sqrt{m_{i} n_{i}} \delta_{m_{i}-1, l_{i}} \delta_{n_{i}-1, k_{i}}\right) \prod_{j \neq i} \delta_{m_{j} l_{j}} \delta_{n_{j} k_{j}} .
\end{aligned}
$$

The (four-dimensional) matrix $\Delta$ represents the quadratic part of the Lagragian. The first difficulty to study the matrix model (3.1) is the computation of its propagator $G$ defined as the inverse of $\Delta$ :

$$
\sum_{r, s \in \mathbb{N}^{D / 2}} \Delta_{m n ; r s} G_{s r ; k l}=\sum_{r, s \in \mathbb{N}^{D / 2}} G_{m n ; r s} \Delta_{s r ; k l}=\delta_{m l} \delta_{n k} .
$$

Fortunately, the action is invariant under $S O(2)^{D / 2}$ thanks to the form (2.1) of the $\Theta$ matrix. It implies a conservation law

$$
\Delta_{m n, k l}=0 \Longleftrightarrow m+k \neq n+l .
$$

The result is $[5,29]$

$$
\begin{aligned}
G_{m, m+h ; l+h, l} & =\frac{\theta}{8 \Omega} \int_{0}^{1} d \alpha \frac{(1-\alpha)^{\frac{\mu_{0}^{2} \theta}{8 \Omega}+\left(\frac{D}{4}-1\right)}}{(1+C \alpha)^{\frac{D}{2}}} \prod_{s=1}^{\frac{D}{2}} G_{m^{s}, m^{s}+h^{s} ; l^{s}+h^{s}, l^{s}}^{(\alpha)} \\
G_{m, m+h ; l+h, l}^{(\alpha)} & =\left(\frac{\sqrt{1-\alpha}}{1+C \alpha}\right)^{m+l+h} \sum_{u=\max (0,-h)}^{\min (m, l)} \mathcal{A}(m, l, h, u)\left(\frac{C \alpha(1+\Omega)}{\sqrt{1-\alpha}(1-\Omega)}\right)^{m+l-2 u}
\end{aligned}
$$

where $\mathcal{A}(m, l, h, u)=\sqrt{\left(\begin{array}{c}m \\ m-u\end{array}\right)\left(\begin{array}{c}m+h \\ m-u\end{array}\right)\left(\begin{array}{c}l \\ l-u\end{array}\right)\left(\begin{array}{c}l+h \\ l-u\end{array}\right)}$ and $C$ is a function in $\Omega: C(\Omega)=\frac{(1-\Omega)^{2}}{4 \Omega}$. The main advantage of the matrix basis is that it simplifies the interaction part: $\phi^{\star 4}$ becomes $\operatorname{Tr} \phi^{4}$. But the propagator becomes very compllicated.

Let us remark that the matrix model (3.1) is dynamical: its quadratic part is not trivial. Usually, matrix models are local. 
Definition 3.1. A matrix model is called local if $G_{m n ; k l}=G(m, n) \delta_{m l} \delta_{n k}$ and non-local otherwise.

In the matrix theories, the Feynman graphs are ribbon graphs. The propagator $G_{m n ; k l}$ is then represented by the Figure 1. In a local matrix model, the propagator preserves

$$
n=\frac{m+h \longleftrightarrow k=l}{\stackrel{\longrightarrow}{m}}+h
$$

Figure 1: Matrix Propagator

the index values along the trajectories (simple lines).

\subsubsection{Topology of ribbon graphs}

The power counting of a matrix model depends on the topological data of its graphs. The figure 2 gives two examples of ribbon graphs. Each ribbon graph may be drawn on a

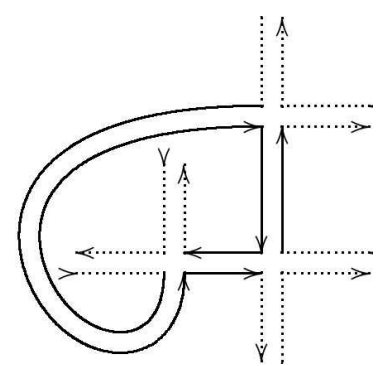

(a) Planar

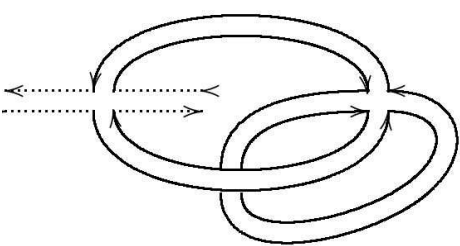

(b) Non-planar

Figure 2: Ribbon Graphs

two-dimensional manifold. Actually each graph defines the surface on which it is drawn. Let a graph $G$ with $V$ vertices, $I$ internal propagators (double lines) and $F$ faces (made of simple lines). The Euler characteristic

$$
\chi=2-2 g=V-I+F
$$

gives the genus $g$ of the manifold. One can make this clear by passing to the dual graph. The dual of a given graph $G$ is obtained by exchanging faces and vertices. The dual graphs of the $\Phi^{4}$ theory are tesselations of the surfaces on which they are drawn. Moreover each direct face broken by exernal legs becomes, in the dual graph, a puncture. If among the $F$ faces of a graph, $B$ are broken, this graph may be drawn on a surface of genus $g=1-\frac{1}{2}(V-I+F)$ with $B$ punctures. The figure 3 gives the topological data of the graphs of the figure 2 .

\subsection{Multi-scale analysis}

In [7], V. R., R. Wulkenhaar and F. V.-T. used the multi-scale analysis to reprove the power counting of the non-commutative $\Phi^{4}$ theory. 

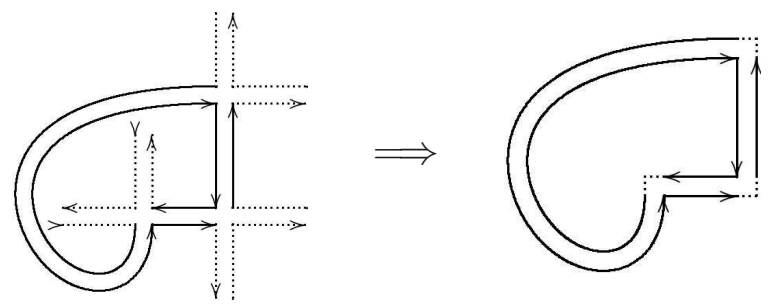

$\left.\begin{array}{l}\mathrm{V}=3 \\ \mathrm{I}=3 \\ \mathrm{~F}=2 \\ \mathrm{~B}=2\end{array}\right\} \Longrightarrow g=0$
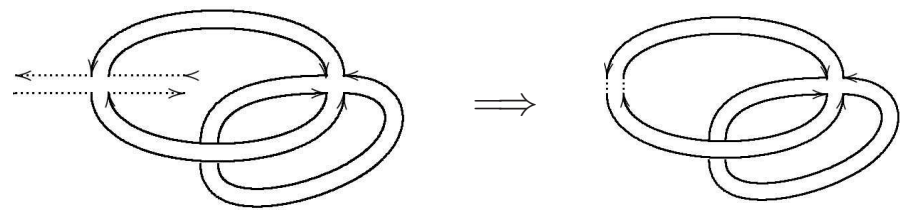

$$
\left.\begin{array}{l}
\mathrm{V}=2 \\
\mathrm{I}=3 \\
\mathrm{~F}=1 \\
\mathrm{~B}=1
\end{array}\right\} \Longrightarrow g=1
$$

Figure 3: Topological Data of Ribbon Graphs

\subsubsection{Bounds on the propagator}

Let $G$ a ribbon graph of the $\Phi_{4}^{4}$ theory with $N$ external legs, $V$ vertices, $I$ internal lines and $F$ faces. Its genus is then $g=1-\frac{1}{2}(V-I+F)$. Four indices $\{m, n ; k, l\} \in \mathbb{N}^{2}$ are associated to each internal line of the graph and two indices to each external line, that is to say $4 I+2 N=8 \mathrm{~V}$ indices. But, at each vertex, the left index of a ribbon equals the right one of the neighbour ribbon. This gives rise to $4 V$ independant identifications which allows to write each index in terms of a set $\mathcal{I}$ made of $4 \mathrm{~V}$ indices, four per vertex, for example the left index of each half-ribbon.

The graph amplitude is then

$$
A_{G}=\sum_{\mathcal{I}} \prod_{\delta \in G} G_{m_{\delta}(\mathcal{I}), n_{\delta}(\mathcal{I}) ; k_{\delta}(\mathcal{I}), l_{\delta}(\mathcal{I})} \delta_{m_{\delta}-l_{\delta}, n_{\delta}-k_{\delta}},
$$

where the four indices of the propagator $G$ of the line $\delta$ are function of $\mathcal{I}$ and written $\left\{m_{\delta}(\mathcal{I}), n_{\delta}(\mathcal{I}) ; k_{\delta}(\mathcal{I}), l_{\delta}(\mathcal{I})\right\}$. We decompose each propagator, given by $(3.5)$ :

$$
G=\sum_{i=0}^{\infty} G^{i} \quad \text { thanks to } \int_{0}^{1} d \alpha=\sum_{i=1}^{\infty} \int_{M^{-2 i}}^{M^{-2(i-1)}} d \alpha, M>1 .
$$

We have an associated decomposition for each amplitude

$$
\begin{aligned}
A_{G} & =\sum_{\mu} A_{G, \mu} \\
A_{G, \mu} & =\sum_{\mathcal{I}} \prod_{\delta \in G} G_{m_{\delta}(\mathcal{I}), n_{\delta}(\mathcal{I}) ; k_{\delta}(\mathcal{I}), l_{\delta}(\mathcal{I})}^{i_{\delta}} \delta_{m_{\delta}(\mathcal{I})-l_{\delta}(\mathcal{I}), n_{\delta}(\mathcal{I})-k_{\delta}(\mathcal{I})}
\end{aligned}
$$

where $\mu=\left\{i_{\delta}\right\}$ runs over the all possible assignements of a positive integer $i_{\delta}$ to each line $\delta$. We proved the following four propositions:

Proposition 3.1. For $M$ large enough, there exists a constant $K$ such that, for $\Omega \in$ $[0.5,1]$, we have the uniform bound

$$
G_{m, m+h ; l+h, l}^{i} \leqslant K M^{-2 i} e^{-\frac{\Omega}{3} M^{-2 i}\|m+l+h\|} .
$$


Proposition 3.2. For $M$ large enough, there exists two constants $K$ and $K_{1}$ such that, for $\Omega \in[0.5,1]$, we have the uniform bound

$$
\begin{aligned}
& G_{m, m+h ; l+h, l}^{i} \\
& \leqslant K M^{-2 i} e^{-\frac{\Omega}{4} M^{-2 i}\|m+l+h\|} \prod_{s=1}^{\frac{D}{2}} \min \left(1,\left(\frac{K_{1} \min \left(m^{s}, l^{s}, m^{s}+h^{s}, l^{s}+h^{s}\right)}{M^{2 i}}\right)^{\frac{\left|m^{s}-l^{s}\right|}{2}}\right) .
\end{aligned}
$$

This bound allows to prove that the only diverging graphs have either a constant index along the trajectories or a total jump of 2 .

Proposition 3.3. For $M$ large enough, there exists a constant $K$ such that, for $\Omega \in\left[\frac{2}{3}, 1\right]$, we have the uniform bound

$$
\sum_{l=-m}^{p} G_{m, p-l, p, m+l}^{i} \leqslant K M^{-2 i} e^{-\frac{\Omega}{4} M^{-2 i}(\|p\|+\|m\|)} .
$$

This bound shows that the propagator is almost local in the following sense: with $m$ fixed, the sum over $l$ doesn't cost anything (see Figure 1). Nevertheless the sums we'll have to perform are entangled (a given index may enter different propagators) so that we need the following proposition.

Proposition 3.4. For $M$ large enough, there exists a constant $K$ such that, for $\Omega \in\left[\frac{2}{3}, 1\right]$, we have the uniform bound

$$
\sum_{l=-m}^{\infty} \max _{p \geqslant \max (l, 0)} G_{m, p-l ; p, m+l}^{i} \leqslant K M^{-2 i} e^{-\frac{\Omega}{36} M^{-2 i}\|m\|} .
$$

We refer to [7] for the proofs of these four propositions.

\subsubsection{Power counting}

About half of the $4 V$ indices initially associated to a graph is determined by the external indices and the delta functions in (3.7). The other indices are summation indices. The power counting consists in finding which sums cost $M^{2 i}$ and which cost $\mathcal{O}(1)$ thanks to (3.13). The $M^{2 i}$ factor comes from (3.11) after a summation over an index $m \in \mathbb{N}^{2}$,

$$
\sum_{m^{1}, m^{2}=0}^{\infty} e^{-c M^{-2 i}\left(m^{1}+m^{2}\right)}=\frac{1}{\left(1-e^{-c M^{-2 i}}\right)^{2}}=\frac{M^{4 i}}{c^{2}}\left(1+\mathcal{O}\left(M^{-2 i}\right)\right)
$$

We first use the delta functions as much as possible to reduce the set $\mathcal{I}$ to a true minimal set $\mathcal{I}^{\prime}$ of independant indices. For this, it is convenient to use the dual graphs where the resolution of the delta functions is equivalent to a usual momentum routing.

\footnotetext{
${ }^{3}$ Recall that each index is in fact made of two indices, one for each symplectic pair of $\mathbb{R}_{\theta}^{4}$.
} 
The dual graph is made of the same propagators than the direct graph except the position of their indices. Whereas in the original graph we have $G_{m n ; k l}=\frac{n}{\rightleftarrows} \rightleftarrows$, the position of the indices in a dual propagator is

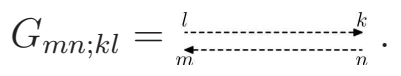

The conservation $\delta_{l-m,-(n-k)}$ in (3.7) implies that the difference $l-m$ is conserved along the propagator. These differences behave like an angular momentum and the conservation of the differences $\ell=l-m$ and $-\ell=n-k$ is nothing else than the conservation of the angular momentum thanks to the symmetry $S O(2) \times S O(2)$ of the action (3.1):

$$
\underset{m}{\stackrel{l}{\longrightarrow} \longleftrightarrow_{-\delta l \longleftarrow \delta l}} \quad l=m+\ell, \quad n=k+(-\ell) .
$$

The cyclicity of the vertices implies the vanishing of the sum of the angular momenta entering a vertex. Thus the angular momentum in the dual graph behaves exactly like the usual momentum in ordinary Feynman graphs.

We know that the number of independent momenta is exactly the number $L^{\prime}$ (= $I-V^{\prime}+1$ for a connected graph) of loops in the dual graph. Each index at a (dual) vertex is then given by a unique reference index and a sum of momenta. If the dual vertex under consideration is an external one, we choose an external index for the reference index. The reference indices in the dual graph correspond to the loop indices in the direct graph. The number of summation indices is then $V^{\prime}-B+L^{\prime}=I+(1-B)$ where $B \geqslant 0$ is the number of broken faces of the direct graph or the number of external vertices in the dual graph.

By using a well-chosen order on the lines, an optimized tree and a $L^{1}-L^{\infty}$ bound, one can prove that the summation over the angular momenta does not cost anything thanks to (3.13). Recall that a connected component is a subgraph for which all internal lines have indices greater than all its external ones. The power counting is then:

$$
\begin{aligned}
A_{G} & \leqslant K^{\prime V} \sum_{\mu} \prod_{i, k} M^{\omega\left(G_{k}^{i}\right)} \\
\text { with } \omega\left(G_{k}^{i}\right) & =4\left(V_{i, k}^{\prime}-B_{i, k}\right)-2 I_{i, k}=4\left(F_{i, k}-B_{i, k}\right)-2 I_{i, k} \\
& =\left(4-N_{i, k}\right)-4\left(2 g_{i, k}+B_{i, k}-1\right)
\end{aligned}
$$

and $N_{i, k}, V_{i, k}, I_{i, k}=2 V_{i, k}-\frac{N_{i, k}}{2}, F_{i, k}$ and $B_{i, k}$ are respectively the numbers of external legs, of vertices, of (internal) propagators, of faces and broken faces of the connected component $G_{k}^{i} ; g_{i, k}=1-\frac{1}{2}\left(V_{i, k}-I_{i, k}+F_{i, k}\right)$ is its genus. We have

Theorem 3.5. The sum over the scales attributions $\mu$ converges if $\forall i, k, \omega\left(G_{k}^{i}\right)<0$.

We recover the power counting obtained in [4].

From this point on, renormalisability of $\phi_{4}^{4}$ can proceed (however remark that it remains limited to $\Omega \in[0.5,1]$ by the technical estimates such as (3.11); this limitation is overcome in the direct space method below).

The multiscale analysis allows to define the so-called effective expansion, in between the bare and the renormalized expansion, which is optimal, both for physical and for 
constructive purposes [34]. In this effective expansion only the subcontributions with all internal scales higher than all external scales have to be renormalised by counterterms of the form of the initial Lagrangian.

In fact only planar such subcontributions with a single external face must be renormalised by such counterterms. This follows simply from the the Grosse-Wulkenhaar moves defined in [4]. These moves translate the external legs along the outer border of the planar graph, up to irrelevant corrections, until they all merge together into a term of the proper Moyal form, which is then absorbed in the effective constants definition. This requires only the estimates (3.11)-(3.14), which were checked numerically in [4].

In this way the relevant and marginal counterterms can be shown to be of the Moyal type, namely renormalise the parameters $\lambda, m$ and $\Omega^{4}$.

Notice that in the multiscale analysis there is no need for the relatively complicated use of Polchinski's equation [39] made in [4]. Polchinski's method, although undoubtedly very elegant for proving perturbative renormalisability does not seem directly suited to constructive purposes, even in the case of simple Fermionic models such as the commutative Gross Neveu model, see e.g. [40].

The BPHZ theorem itself for the renormalised expansion follows from finiteness of the effective expansion by developing the counterterms still hidden in the effective couplings. Its own finiteness can be checked e.g. through the standard classification of forests [34]. Let us however recall once again that in our opinion the effective expansion, not the renormalised one is the more fundamental object, both to describe the physics and to attack deeper mathematical problems, such as those of constructive theory [34, 41].

The matrix base simplfies very much at $\Omega=1$, where the matrix propagator becomes diagonal, i.e. conserves exactly indices. This property has been used for the general proof that the beta function of the theory vanishes in the ultraviolet regime [24], leading to the exciting perspective of a full non-perturbative construction of the model.

\subsection{Propagators on non-commutative space}

We give here the results we get in [20]. In this article, we computed the $x$-space and matrix basis kernels of operators which generalize the Mehler kernel (2.34). Then we proceeded to a study of the scaling behaviours of these kernels in the matrix basis. This work is useful to study the non-commutative Gross-Neveu model in the matrix basis.

\subsubsection{Bosonic kernel}

The following lemma generalizes the Mehler kernel [28]:

Lemma 3.6. Let $H$ the operator:

$$
H=\frac{1}{2}\left(-\Delta+\Omega^{2} x^{2}-2 \imath B\left(x_{0} \partial_{1}-x_{1} \partial_{0}\right)\right) .
$$

The $x$-space kernel of $e^{-t H}$ is:

$$
e^{-t H}\left(x, x^{\prime}\right)=\frac{\Omega}{2 \pi \sinh \Omega t} e^{-A},
$$

\footnotetext{
${ }^{4}$ The wave function renormalisation i.e. renormalisation of the $\partial_{\mu} \phi \star \partial^{\mu} \phi$ term can be absorbed in a rescaling of the field, called "field strength renromalization."
} 


$$
A=\frac{\Omega \cosh \Omega t}{2 \sinh \Omega t}\left(x^{2}+x^{\prime 2}\right)-\frac{\Omega \cosh B t}{\sinh \Omega t} x \cdot x^{\prime}-\imath \frac{\Omega \sinh B t}{\sinh \Omega t} x \wedge x^{\prime} .
$$

Remark. The Mehler kernel corresponds to $B=0$. The limit $\Omega=B \rightarrow 0$ gives the usual heat kernel.

Lemma 3.7. Let $H$ be given by (3.20) with $\Omega(B) \rightarrow 2 \Omega / \theta(2 B \theta)$. Its inverse in the matrix basis is:

$$
\begin{gathered}
H_{m, m+h ; l+h, l}^{-1}=\frac{\theta}{8 \Omega} \int_{0}^{1} d \alpha \frac{(1-\alpha)^{\frac{\mu_{0}^{2} \theta}{8 \Omega}+\left(\frac{D}{4}-1\right)}}{(1+C \alpha)^{\frac{D}{2}}}(1-\alpha)^{-\frac{4 B}{8 \Omega} h} \prod_{s=1}^{\frac{D}{2}} G_{m^{s}, m^{s}+h^{s} ; l^{s}+h^{s}, l^{s}}^{(\alpha)} \\
G_{m, m+h ; l+h, l}^{(\alpha)}=\left(\frac{\sqrt{1-\alpha}}{1+C \alpha}\right)^{m+l+h} \sum_{u=\max (0,-h)}^{\min (m, l)} \mathcal{A}(m, l, h, u)\left(\frac{C \alpha(1+\Omega)}{\sqrt{1-\alpha}(1-\Omega)}\right)^{m+l-2 u}
\end{gathered}
$$

where $\mathcal{A}(m, l, h, u)=\sqrt{\left(\begin{array}{c}m \\ m-u\end{array}\right)\left(\begin{array}{c}m+h \\ m-u\end{array}\right)\left(\begin{array}{c}l \\ l-u\end{array}\right)\left(\begin{array}{l}l+h \\ l-u\end{array}\right)}$ and $C$ is a function of $\Omega: C(\Omega)=\frac{(1-\Omega)^{2}}{4 \Omega}$.

\subsubsection{Fermionic kernel}

On the Moyal space, we modified the commutative Gross-Neveu model by adding a $\not x$ term (see lemma 2.4). We have

$$
\begin{aligned}
G(x, y)= & -\frac{\Omega}{\theta \pi} \int_{0}^{\infty} \frac{d t}{\sinh (2 \widetilde{\Omega} t)} e^{-\frac{\widetilde{\Omega}}{2} \operatorname{coth}(2 \widetilde{\Omega} t)(x-y)^{2}+\imath \widetilde{\Omega} x \wedge y} \\
& \{\imath \widetilde{\Omega} \operatorname{coth}(2 \widetilde{\Omega} t)(\not x-y)+\Omega(\not{x}-\widetilde{y})-\mu\} e^{-2 \imath \widetilde{\Omega} t \gamma^{0} \gamma^{1}} e^{-t \mu^{2}} .
\end{aligned}
$$

It will be useful to express $G$ in terms of commutators:

$$
\begin{aligned}
G(x, y)= & -\frac{\Omega}{\theta \pi} \int_{0}^{\infty} d t\left\{\imath \widetilde{\Omega} \operatorname{coth}(2 \widetilde{\Omega} t)\left[\not x, \Gamma^{t}\right](x, y)\right. \\
& \left.+\Omega\left[\not{t}, \Gamma^{t}\right](x, y)-\mu \Gamma^{t}(x, y)\right\} e^{-2 \imath \widetilde{\Omega} t \gamma^{0} \gamma^{1}} e^{-t \mu^{2}},
\end{aligned}
$$

where

$$
\Gamma^{t}(x, y)=\frac{1}{\sinh (2 \widetilde{\Omega} t)} e^{-\frac{\widetilde{\Omega}}{2} \operatorname{coth}(2 \widetilde{\Omega} t)(x-y)^{2}+\imath \widetilde{\Omega} x \wedge y}
$$

with $\widetilde{\Omega}=\frac{2 \Omega}{\theta}$ and $x \wedge y=x^{0} y^{1}-x^{1} y^{0}$.

We now give the expression of the Fermionic kernel (3.25) in the matrix basis. The inverse of the quadratic form

$$
\Delta=p^{2}+\mu^{2}+\frac{4 \Omega^{2}}{\theta^{2}} x^{2}+\frac{4 B}{\theta} L_{2}
$$


is given by (3.23) in the preceeding section:

$$
\begin{aligned}
\Gamma_{m, m+h ; l+h, l} & =\frac{\theta}{8 \Omega} \int_{0}^{1} d \alpha \frac{(1-\alpha)^{\frac{\mu^{2} \theta}{8 \Omega}-\frac{1}{2}}}{(1+C \alpha)} \Gamma_{m, m+h ; l+h, l}^{\alpha} \\
\Gamma_{m, m+h ; l+h, l}^{(\alpha)} & =\left(\frac{\sqrt{1-\alpha}}{1+C \alpha}\right)^{m+l+h}(1-\alpha)^{-\frac{B h}{2 \Omega}} \\
& \sum_{u=0}^{\min (m, l)} \mathcal{A}(m, l, h, u)\left(\frac{C \alpha(1+\Omega)}{\sqrt{1-\alpha}(1-\Omega)}\right)^{m+l-2 u} .
\end{aligned}
$$

The Fermionic propagator $G$ (3.25) in the matrix basis may be deduced from the kernel (3.28). We just set $B=\Omega$, add the missing term with $\gamma^{0} \gamma^{1}$ and compute the action of $-\not p-\Omega * x+\mu$ on $\Gamma$. We must then evaluate $\left[x^{\nu}, \Gamma\right]$ in the matrix basis:

$$
\begin{aligned}
{\left[x^{0}, \Gamma\right]_{m, n ; k, l}=} & \pi \theta \sqrt{\frac{\theta}{8}}\left\{\sqrt{m+1} \Gamma_{m+1, n ; k, l}-\sqrt{l} \Gamma_{m, n ; k, l-1}+\sqrt{m} \Gamma_{m-1, n ; k, l}\right. \\
& -\sqrt{l+1} \Gamma_{m, n ; k, l+1}+\sqrt{n+1} \Gamma_{m, n+1 ; k, l}-\sqrt{k} \Gamma_{m, n ; k-1, l} \\
& \left.+\sqrt{n} \Gamma_{m, n-1 ; k, l}-\sqrt{k+1} \Gamma_{m, n ; k+1, l}\right\}, \\
{\left[x^{1}, \Gamma\right]_{m, n ; k, l}=} & 2 \imath \pi \theta \sqrt{\frac{\theta}{8}}\left\{\sqrt{m+1} \Gamma_{m+1, n ; k, l}-\sqrt{l} \Gamma_{m, n ; k, l-1}-\sqrt{m} \Gamma_{m-1, n ; k, l}\right. \\
& +\sqrt{l+1} \Gamma_{m, n ; k, l+1}-\sqrt{n+1} \Gamma_{m, n+1 ; k, l}+\sqrt{k} \Gamma_{m, n ; k-1, l} \\
& \left.+\sqrt{n} \Gamma_{m, n-1 ; k, l}-\sqrt{k+1} \Gamma_{m, n ; k+1, l}\right\} .
\end{aligned}
$$

This allows to prove:

Lemma 3.8. Let $G_{m, n ; k, l}$ the kernel, in the matrix basis, of the operator $(\not p+\Omega \not c+\mu)^{-1}$. We have:

$$
\begin{aligned}
G_{m, n ; k, l}= & -\frac{2 \Omega}{\theta^{2} \pi^{2}} \int_{0}^{1} d \alpha G_{m, n ; k, l}^{\alpha}, \\
G_{m, n ; k, l}^{\alpha}= & \left(\imath \widetilde{\Omega} \frac{2-\alpha}{\alpha}\left[\not x, \Gamma^{\alpha}\right]_{m, n ; k, l}+\Omega\left[\not x, \Gamma^{\alpha}\right]_{m, n ; k, l}-\mu \Gamma_{m, n ; k, l}^{\alpha}\right) \\
& \times\left(\frac{2-\alpha}{2 \sqrt{1-\alpha}} \mathbb{1}_{2}-\imath \frac{\alpha}{2 \sqrt{1-\alpha}} \gamma^{0} \gamma^{1}\right) .
\end{aligned}
$$

where $\Gamma^{\alpha}$ is given by (3.29) and the commutators bu the formulas (3.30) and (3.31).

The first two terms in the equation (3.33) contain commutators and will be gathered under the name $G_{m, n ; k, l}^{\alpha, \text { comm }}$. The last term will be called $G_{m, n ; k, l}^{\alpha, \text { mass }}$ :

$$
\begin{aligned}
G_{m, n ; k, l}^{\alpha, \text { comm }}= & \left(\imath \widetilde{\Omega} \frac{2-\alpha}{\alpha}\left[\not x, \Gamma^{\alpha}\right]_{m, n ; k, l}+\Omega\left[\not \Gamma^{\alpha}\right]_{m, n ; k, l}\right) \\
& \times\left(\frac{2-\alpha}{2 \sqrt{1-\alpha}} \mathbb{1}_{2}-\imath \frac{\alpha}{2 \sqrt{1-\alpha}} \gamma^{0} \gamma^{1}\right), \\
G_{m, n ; k, l}^{\alpha, \text { mass }}= & -\mu \Gamma_{m, n ; k, l}^{\alpha} \times\left(\frac{2-\alpha}{2 \sqrt{1-\alpha}} \mathbb{1}_{2}-\imath \frac{\alpha}{2 \sqrt{1-\alpha}} \gamma^{0} \gamma^{1}\right) .
\end{aligned}
$$




\subsubsection{Bounds}

We use the multi-scale analysis to study the behaviour of the propagator (3.33) and revisit more finely the bounds (3.11) to (3.14). In a slice $i$, the propagator is

$$
\begin{gathered}
\Gamma_{m, m+h, l+h, l}^{i}=\frac{\theta}{8 \Omega} \int_{M^{-2 i}}^{M^{-2(i-1)}} d \alpha \frac{(1-\alpha)^{\frac{\mu_{0}^{2} \theta}{8 \Omega}-\frac{1}{2}}}{(1+C \alpha)} \Gamma_{m, m+h ; l+h, l}^{(\alpha)} . \\
G_{m, n ; k, l}=\sum_{i=1}^{\infty} G_{m, n ; k, l}^{i} ; G_{m, n ; k, l}^{i}=-\frac{2 \Omega}{\theta^{2} \pi^{2}} \int_{M^{-2 i}}^{M^{-2(i-1)}} d \alpha G_{m, n ; k, l}^{\alpha} .
\end{gathered}
$$

Let $h=n-m$ and $p=l-m$. Without loss of generality, we assume $h \geqslant 0$ and $p \geqslant 0$. Then the smallest index among $m, n, k, l$ is $m$ and the biggest is $k=m+h+p$. We have:

Theorem 3.9. Under the assumptions $h=n-m \geqslant 0$ and $p=l-m \geqslant 0$, there exists $K, c \in \mathbb{R}_{+}$(c depends on $\Omega$ ) such that the propagator of the non-commutative Gross-Neveu model in a slice $i$ obeys the bound

$$
\begin{aligned}
\left|G_{m, n ; k, l}^{i, \mathrm{comm}}\right| \leqslant & K M^{-i}\left(\chi(\alpha k>1) \frac{\exp \left\{-\frac{c p^{2}}{1+k M^{-2 i}}-\frac{c M^{-2 i}}{1+k}\left(h-\frac{k}{1+C}\right)^{2}\right\}}{\left(1+\sqrt{k M^{-2 i}}\right)}\right. \\
& \left.+\min \left(1,(\alpha k)^{p}\right) e^{-c k M^{-2 i}-c p}\right) .
\end{aligned}
$$

The mass term is slightly different:

$$
\begin{aligned}
\left|G_{m, n ; k, l}^{i, \text { mass }}\right| \leqslant & K M^{-2 i}\left(\chi(\alpha k>1) \frac{\exp \left\{-\frac{c p^{2}}{1+k M^{-2 i}}-\frac{c M^{-2 i}}{1+k}\left(h-\frac{k}{1+C}\right)^{2}\right\}}{1+\sqrt{k M^{-2 i}}}\right. \\
& \left.+\min \left(1,(\alpha k)^{p}\right) e^{-c k M^{-2 i}-c p}\right) .
\end{aligned}
$$

Remark. We can redo the same analysis for the $\Phi^{4}$ propagator and get

$$
G_{m, n ; k, l}^{i} \leqslant K M^{-2 i} \min \left(1,(\alpha k)^{p}\right) e^{-c\left(M^{-2 i} k+p\right)}
$$

which allows to recover the bounds (3.11) to (3.14).

\subsection{Propagators and renormalisability}

Let us consider the propagator (3.32) of the non-commutative Gross-Neveu model. We saw in section 3.3.3 that there exists two regions in the space of indices where the propagator behaves very differently. In one of them it behaves as the $\Phi^{4}$ propagator and leads then to the same power counting. In the critical region, we have

$$
G^{i} \leqslant K \frac{M^{-i}}{1+\sqrt{k M^{-2 i}}} e^{-\frac{c p^{2}}{1+k M^{-2 i}}-\frac{c M^{-2 i}}{1+k}\left(h-\frac{k}{1+C}\right)^{2}} .
$$

The point is that such a propagator does not allow to sum two reference indices with a unique line. This fact was useful in the proof of the power counting of the $\Phi^{4}$ model. This leads to a renormalisable $\mathrm{UV} / \mathrm{IR}$ mixing. 


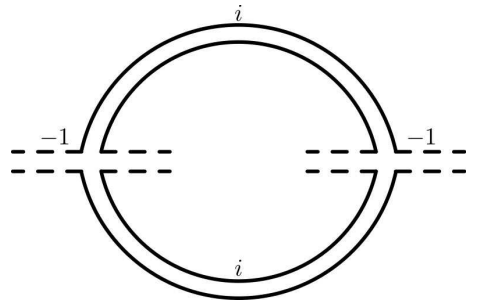

(a) At scale $i$

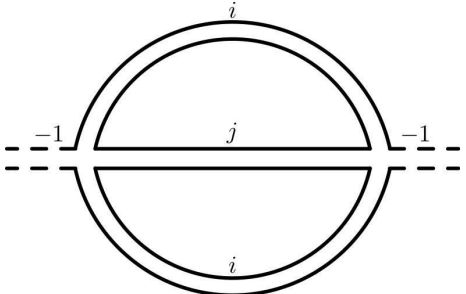

(b) At scale $j$

Figure 4: Sunset Graph

Let us consider the graph in figure $4 \mathrm{~b}$ where the two external lines bear an index $i \gg 1$ and the internal one an index $j<i$. The propagator (3.32) obeys the bound in Prop. (3.13) which means that it is almost local. We only have to sum over one index per internal face.

On the graph of the figure 4a, if the two lines inside are true external ones, the graph has two broken faces and there is no index to sum over. Then by using Prop. (3.11) we get $A_{G} \leqslant M^{-2 i}$. The sum over $i$ converges and we have the same behaviour as the $\Phi^{4}$ theory, that is to say the graphs with $B \geqslant 2$ broken faces are finite. But if these two lines belongs to a line of scale $j<i$ (see figure 4b), the result is different. Indeed, at scale $i$, we recover the graph of figure 4a. To maintain the previous result $\left(M^{-2 i}\right)$, we should sum the two indices corresponding to the internal faces with the propagator of scale $j$. This is not possible. Instead we have:

$$
\sum_{k, h} M^{-2 i-j} e^{-M^{-2 i} k} \frac{e^{-\frac{c M^{-2 j}}{1+k}\left(h-\frac{k}{1+C}\right)^{2}}}{1+\sqrt{k M^{-2 j}}} \leqslant K M^{j} .
$$

The sum over $i$ diverges logarithmically. The graph of figure 4a converges if it is linked to true exernal legs et diverges if it is a subgraph of a graph at a lower scale. The power counting depends on the scales lower than the lowest scale of the graph. It can't then be factorized into the connected components: this is $\mathrm{UV} / \mathrm{IR}$ mixing.

Let's remark that the graph of figure $4 \mathrm{a}$ is not renormalisable by a counter-term in the Lagrangian. Its logarithmic divergence can't be absorbed in a redefinition of a coupling constant. Fortunately the renormalisation of the two-point graph of figure $4 \mathrm{~b}$ makes the four-point subdivergence finite [21]. This makes the non-commutative Gross-Neveu model renormalisable.

\section{Direct space}

We want now to explain how the power counting analysis can be performed in direct space, and the "Moyality" of the necessary counterterms can be checked by a Taylor expansion which is a generalization of the one used in direct commutative space.

In the commutative case there is translation invariance, hence each propagator depends on a single difference variable which is short in the ultraviolet regime; in the non-commutative case the propagator depends both of the difference of end positions, 
which is again short in the uv regime, but also of the sum which is long in the uv regime, considering the explicit form (2.34) of the Mehler kernel.

This distinction between short and long variables is at the basis of the power counting analysis in direct space.

\subsection{Short and long variables}

Let $G$ be an arbitrary connected graph. The amplitude associated with this graph is in direct space (with hopefully self-explaining notations):

$$
\begin{aligned}
A_{G}= & \int_{v, i=1, \ldots 4} d x_{v, i} \prod_{l} d t_{l} \\
& \prod_{v}\left[\delta\left(x_{v, 1}-x_{v, 2}+x_{v, 3}-x_{v, 4}\right) e^{i \sum_{i<j}(-1)^{i+j+1} x_{v, i} \theta^{-1} x_{v, j}}\right] \prod_{l} C_{l}, \\
C_{l}= & \frac{\Omega^{2}}{\left[2 \pi \sinh \left(\Omega t_{l}\right)\right]^{2}} e^{-\frac{\Omega}{2} \operatorname{coth}\left(\Omega t_{l}\right)\left(x_{v, i(l)}^{2}+x_{v^{\prime}, i^{\prime}(l)}^{2}\right)+\frac{\Omega}{\sinh \left(\Omega t_{l}\right)} x_{v, i(l)} \cdot x_{v^{\prime}, i^{\prime}(l)}-\mu_{0}^{2} t_{l}} .
\end{aligned}
$$

For each line $l$ of the graph joining positions $x_{v, i(l)}$ and $x_{v^{\prime}, i^{\prime}(l)}$, we choose an orientation and we define the "short" variable $u_{l}=x_{v, i(l)}-x_{v^{\prime}, i^{\prime}(l)}$ and the "long" variable $v_{l}=$ $x_{v, i(l)}+x_{v^{\prime}, i^{\prime}(l)}$.

With these notations, defining $\Omega t_{l}=\alpha_{l}$, the propagators in our graph can be written as:

$$
\int_{0}^{\infty} \prod_{l} \frac{\Omega d \alpha_{l}}{\left[2 \pi \sinh \left(\alpha_{l}\right)\right]^{2}} e^{-\frac{\Omega}{4} \operatorname{coth}\left(\frac{\alpha_{l}}{2}\right) u_{l}^{2}-\frac{\Omega}{4} \tanh \left(\frac{\alpha_{l}}{2}\right) v_{l}^{2}-\frac{\mu_{0}^{2}}{\Omega} \alpha_{l}} .
$$

As in matrix space we can slice each propagator according to the size of its $\alpha$ parameter and obtain the multiscale represenation of each Feynman amplitude:

$$
\begin{aligned}
A_{G}= & \sum_{\mu} A_{G, \mu} \quad, \quad A_{G, \mu}=\int \prod_{v, i=1, \ldots 4} d x_{v, i} \prod_{l} C_{l}^{i_{\mu}(l)}\left(u_{l}, v_{l}\right) \\
& \prod_{v}\left[\delta\left(x_{v, 1}-x_{v, 2}+x_{v, 3}-x_{v, 4}\right) e^{\imath \sum_{i<j}(-1)^{i+j+1} x_{v, i} \theta^{-1} x_{v, j}}\right] \\
C^{i}(u, v)= & \int_{M^{-2 i}}^{M^{-2(i-1)}} \frac{\Omega d \alpha}{[2 \pi \sinh (\alpha)]^{2}} e^{-\frac{\Omega}{4} \operatorname{coth}\left(\frac{\alpha}{2}\right) u^{2}-\frac{\Omega}{4} \tanh \left(\frac{\alpha}{2}\right) v^{2}-\frac{\mu_{0}^{2}}{\Omega} \alpha},
\end{aligned}
$$

where $\mu$ runs over scales attributions $\left\{i_{\mu}(l)\right\}$ for each line $l$ of the graph, and the sliced propagator $C^{i}$ in slice $i \in \mathbb{N}$ obeys the crude bound:

Lemma 4.1. For some constants $K$ (large) and c (small):

$$
C^{i}(u, v) \leqslant K M^{2 i} e^{-c\left[M^{i}\|u\|+M^{-i}\|v\|\right]}
$$

(which a posteriori justifies the terminology of "long" and "short" variables).

The proof is elementary. 


\subsection{Routing, Filk moves}

\subsubsection{Oriented graphs}

We pick a tree $T$ of lines of the graph, hence connecting all vertices, pick with a root vertex and build an orientation of all the lines of the graph in an inductive way. Starting from an arbitrary orientation of a field at the root of the tree, we climb in the tree and at each vertex of the tree we impose cyclic order to alternate entering and exiting tree lines and loop half-lines, as in figure 5a. Then we look at the loop lines. If every loop lines

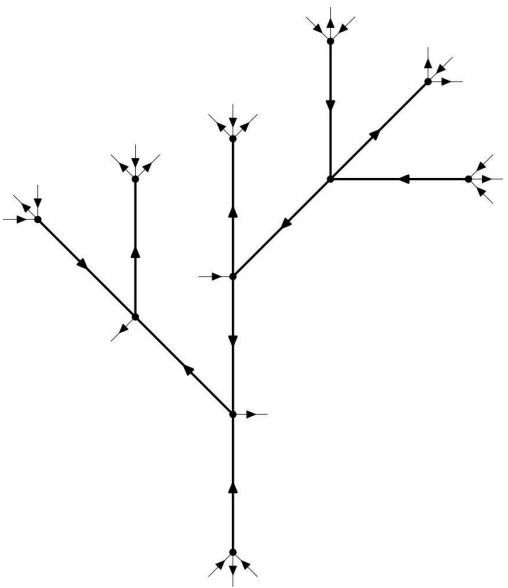

(a) Orientation of a tree

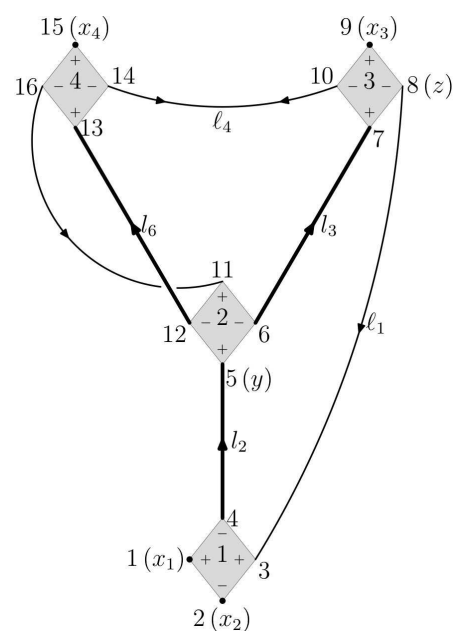

(b) A non-orientable graph

Figure 5: Orientation

consist in the contraction of an entering and an exiting line, the graph is called orientable. Otherwise we call it non-orientable as in figure 5b.

\subsubsection{Position routing}

There are $n \delta$ functions in an amplitude with $n$ vertices, hence $n$ linear equations for the $4 n$ positions, one for each vertex. The position routing associated to the tree $T$ solves this system by passing to another equivalent system of $n$ linear equations, one for each branch of the tree. This is a triangular change of variables, of Jacobian 1. This equivalent system is obtained by summing the arguments of the $\delta$ functions of the vertices in each branch. This change of variables is exactly the $x$-space analog of the resolution of momentum conservation called momentum routing in the standard physics literature of commutative field theory, except that one should now take care of the additional \pm cyclic signs.

One can prove [3] that the rank of the system of $\delta$ functions in an amplitude with $n$ vertices is

- $n-1$ if the graph is orientable

- $n$ if the graph is non-orientable

The position routing change of variables is summarized by the following lemma: 
Lemma 4.2 (Position Routing). We have, calling $I_{G}$ the remaining integrand in (4.3):

$$
\begin{aligned}
A_{G} & =\int\left[\prod_{v}\left[\delta\left(x_{v, 1}-x_{v, 2}+x_{v, 3}-x_{v, 4}\right)\right]\right] I_{G}\left(\left\{x_{v, i}\right\}\right) \\
& =\int \prod_{b} \delta\left(\sum_{l \in T_{b} \cup L_{b}} u_{l}+\sum_{l \in L_{b,+}} v_{l}-\sum_{l \in L_{b,-}} v_{l}+\sum_{f \in X_{b}} \epsilon(f) x_{f}\right) I_{G}\left(\left\{x_{v, i}\right\}\right),
\end{aligned}
$$

where $\epsilon(f)$ is \pm 1 depending on whether the field $f$ enters or exits the branch.

We can now use the system of delta functions to eliminate variables. It is of course better to eliminate long variables as their integration costs a factor $M^{4 i}$ whereas the integration of a short variable brings $M^{-4 i}$. Rough power counting, neglecting all oscillations of the vertices leads therefore, in the case of an orientable graph with $N$ external fields, $n$ internal vertices and $l=2 n-N / 2$ internal lines at scale $i$ to:

- a factor $M^{2 i(2 n-N / 2)}$ coming from the $M^{2 i}$ factors for each line of scale $i$ in (4.5),

- a factor $M^{-4 i(2 n-N / 2)}$ for the $l=2 n-N / 2$ short variables integrations,

- a factor $M^{4 i(n-N / 2+1)}$ for the long variables after eliminating $n-1$ of them using the delta functions.

The total factor is therefore $M^{-(N-4) i}$, the ordinary scaling of $\phi_{4}^{4}$, which means that only two and four point subgraphs $(N \leqslant 4)$ diverge when $i$ has to be summed.

In the non-orientable case, we can eliminate one additional long variable since the rank of the system of delta functions is larger by one unit! Therefore we get a power counting bound $M^{-N i}$, which proves that only orientable graphs may diverge.

In fact we of course know that not all orientable two and four point subgraphs diverge but only the planar ones with a single external face. (It is easy to check that all such planar graphs are indeed orientable).

Since only these planar subgraphs with a single external face can be renormalised by Moyal counterterms, we need to prove that orientable, non-planar graphs or orientable planar graphs with several external faces have in fact a better power counting than this crude estimate. This can be done only by exploiting their vertices oscillations. We explain now how to do this with minimal effort.

\subsubsection{Filk moves and rosettes}

Following Filk [8], we can contract all lines of a spanning tree $T$ and reduce $G$ to a single vertex with "tadpole loops" called a "rosette graph". This rosette is a cycle (which is the border of the former tree) bearing loops lines on it (see figure 6): Remark that the rosette can also be considered as a big vertex, with $r=2 n+2$ fields, on which $N$ are external fields with external variables $x$ and $2 n+2-N$ are loop fields for the corresponding $n+1-N / 2$ loops. When the graph is orientable, the rosette is also orientable, which means that turning around the rosette the lines alternatively enter and exit. These lines correspond to the contraction of the fields on the border of the tree $T$ before the Filk contraction, also called the "first Filk move". 


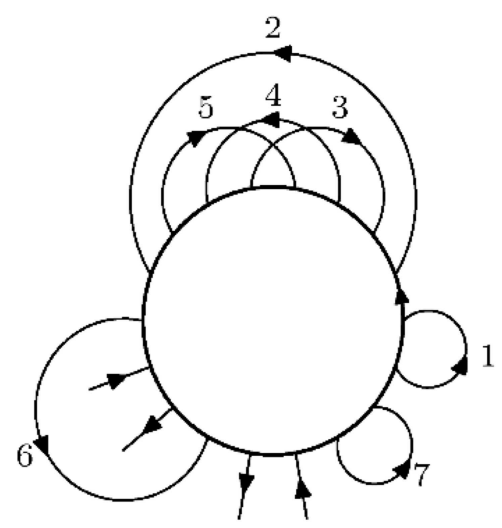

Figure 6: A rosette

\subsubsection{Rosette factor}

We start from the root and turn around the tree in the trigonometrical sense. We number separately all the fields as $1, \ldots, 2 n+2$ and all the tree lines as $1, \ldots, n-1$ in the order they are met.

Lemma 4.3. The rosette contribution after a complete first Filk reduction is exactly:

$$
\delta\left(v_{1}-v_{2}+\cdots-v_{2 n+2}+\sum_{l \in T} u_{l}\right) e^{i V Q V+i U R U+i U S V}
$$

where the $v$ variables are the long or external variables of the rosette, counted with their signs, and the quadratic oscillations for these variables is

$$
V Q V=\sum_{0 \leqslant i<j \leqslant r}(-1)^{i+j+1} v_{i} \theta^{-1} v_{j}
$$

We have now to analyze in detail this quadratic oscillation of the remaining long loop variables since it is essential to improve power counting. We can neglect the secondary oscillations $U R U$ and $U S V$ which imply short variables.

The second Filk reduction [8] further simplifies the rosette factor by erasing the loops of the rosette which do not cross any other loops or arch over external fields. It can be shown that the loops which disappear in this operation correspond to those long variables who do not appear in the quadratic form $Q$.

Using the remaining oscillating factors one can prove that non-planar graphs with genus larger than one or with more than one external face do not diverge.

The basic mechanism to improve the power counting of a single non-planar subgraph is the following:

$$
\begin{aligned}
& \int d w_{1} d w_{2} e^{-M^{-2 i_{1}} w_{1}^{2}-M^{-2 i_{2}} w_{2}^{2}-i w_{1} \theta^{-1} w_{2}+w_{1} \cdot E_{1}(x, u)+w_{2} E_{2}(x, u)} \\
= & \int d w_{1}^{\prime} d w_{2}^{\prime} e^{-M^{-2 i_{1}}\left(w_{1}^{\prime}\right)^{2}-M^{-2 i_{2}}\left(w_{2}^{\prime}\right)^{2}+i w_{1}^{\prime} \theta^{-1} w_{2}^{\prime}+(u, x) Q(u, x)} \\
= & K M^{4 i_{1}} \int d w_{2}^{\prime} e^{-\left(M^{2 i_{1}}+M^{-2 i_{2}}\right)\left(w_{2}^{\prime}\right)^{2}}=K M^{4 i_{1}} M^{-4 i_{2}} .
\end{aligned}
$$


In these equations we used for simplicity $M^{-2 i}$ instead of the correct but more complicated factor $(\Omega / 4) \tanh (\alpha / 2)$ (of course this does not change the argument) and we performed a unitary linear change of variables $w_{1}^{\prime}=w_{1}+\ell_{1}(x, u), w_{2}^{\prime}=w_{2}+\ell_{2}(x, u)$ to compute the oscillating $w_{1}^{\prime}$ integral. The gain in (4.9) is $M^{-8 i_{2}}$, which is the difference between $M^{-4 i_{2}}$ and the normal factor $M^{4 i_{2}}$ that the $w_{2}$ integral would have cost if we had done it with the regular $e^{-M^{-2 i_{2}} w_{2}^{2}}$ factor for long variables. To maximize this gain we can assume $i_{1} \leqslant i_{2}$.

This basic argument must then be generalized to each non-planar subgraph in the multiscale analysis, which is possible.

Finally it remains to consider the case of subgraphs which are planar orientable but with more than one external face. In that case there are no crossing loops in the rosette but there must be at least one loop line arching over a non trivial subset of external legs (see e.g. line 6 in figure 6). We have then a non trivial integration over at least one external variable, called $x$, of at least one long loop variable called $w$. This "external" $x$ variable without the oscillation improvement would be integrated with a test function of scale 1 (if it is a true external line of scale 1) or better (if it is a higher long loop variable) ${ }^{5}$. But we get now

$$
\begin{aligned}
& \int d x d w e^{-M^{-2 i} w^{2}-i w \theta^{-1} x+w \cdot E_{1}\left(x^{\prime}, u\right)} \\
= & K M^{4 i} \int d x e^{-M^{+2 i} x^{2}}=K^{\prime},
\end{aligned}
$$

so that a factor $M^{4 i}$ in the former bound becomes $\mathcal{O}(1)$ hence is improved by $M^{-4 i}$.

In this way we can reduce the convergence of the multiscale analysis to the problem of renormalisation of planar two- and four-point subgraphs with a single external face, which we treat in the next section.

Remark that the power counting obtained in this way is still not optimal. To get the same level of precision than with the matrix base requires e.g. to display $g$ independent improvements of the type (4.9) for a graph of genus $g$. This is doable but basically requires a reduction of the quadratic form $Q$ for single-faced rosette (also called "hyperrosette") into $g$ standard symplectic blocks through the so-called "third Filk move" introduced in [19]. We return to this question in section 4.4.

\subsection{Renormalisation}

\subsubsection{Four-point function}

Consider the amplitude of a four-point graph $G$ which in the multiscale expansion has all its internal scales higher than its four external scales.

The idea is that one should compare its amplitude to a similar amplitude with a "Moyal factor" $\exp \left(2 \imath \theta^{-1}\left(x_{1} \wedge x_{2}+x_{3} \wedge x_{4}\right)\right) \delta(\Delta)$ factorized in front, where $\Delta=x_{1}-x_{2}+x_{3}-$ $x_{4}$. But precisely because the graph is planar with a single external face we understand that the external positions $x$ only couple to short variables $U$ of the internal amplitudes through the global delta function and the oscillations. Hence we can break this coupling

\footnotetext{
${ }^{5}$ Since the loop line arches over a non trivial (i.e. neither full nor empty) subset of external legs of the rosette, the variable $x$ cannot be the full combination of external variables in the "root" $\delta$ function.
} 
by a systematic Taylor expansion to first order. This separates a piece proportional to "Moyal factor", then absorbed into the effective coupling constant, and a remainder which has at least one additional small factor which gives him improved power counting.

This is done by expressing the amplitude for a graph with $N=4, g=0$ and $B=1$ as:

$$
\begin{aligned}
A(G)\left(x_{1}, x_{2}, x_{3}, x_{4}\right)= & \int \exp \left(2 \imath \theta^{-1}\left(x_{1} \wedge x_{2}+x_{3} \wedge x_{4}\right)\right) \prod_{\ell \in T_{k}^{i}} d u_{\ell} C_{\ell}\left(u_{\ell}, U_{\ell}, V_{\ell}\right) \\
& {\left[\prod_{l \in G_{k}^{i} l \notin T} d u_{l} d v_{l} C_{l}\left(u_{l}, v_{l}\right)\right] e^{\imath U R U+\imath U S V} } \\
& \left\{\delta(\Delta)+\int_{0}^{1} d t\left[\mathfrak{U} \cdot \nabla \delta(\Delta+t \mathfrak{U})+\delta(\Delta+t \mathfrak{U})\left[\imath X Q U+\mathfrak{R}^{\prime}(t)\right]\right] e^{\imath t X Q U+\mathfrak{R}(t)}\right\} .
\end{aligned}
$$

where $C_{\ell}\left(u_{\ell}, U_{\ell}, V_{\ell}\right)$ is the propagator taken at $X_{\ell}=0, \mathfrak{U}=\sum_{\ell} u_{\ell}$ and $\mathfrak{R}(t)$ is a correcting term involving tanh $\alpha_{\ell}[X . X+X .(U+V)]$.

The first term is of the initial $\int \operatorname{Tr} \phi \star \phi \star \phi \star \phi$ form. The rest no longer diverges, since the $U$ and $\mathfrak{R}$ provide the necessary small factors.

\subsubsection{Two-point function}

Following the same strategy we have to Taylor-expand the coupling between external variables and $U$ factors in two point planar graphs with a single external face to third order and some non-trivial symmetrization of the terms acording to the two external arguments to cancel some odd contributions. The corresponding factorized relevant and marginal contributions can be then shown to give rise only to

- A mass counterterm,

- A wave function counterterm,

- An harmonic potential counterterm.

and the remainder has convergent power counting. This concludes the construction of the effective expansion in this direct space multiscale analysis.

Again the BPHZ theorem itself for the renormalised expansion follows by developing the counterterms still hidden in the effective couplings and its finiteness follows from the standard classification of forests. See however the remarks at the end of section 3.2.2.

Since the bound (4.5) works for any $\Omega \neq 0$, an additional bonus of the $x$-space method is that it proves renormalisability of the model for any $\Omega$ in $] 0,1]^{6}$, whether the matrix method proved it only for $\Omega$ in $] 0.5,1]$.

\footnotetext{
${ }^{6}$ The case $\Omega$ in $[1,+\infty[$ is irrelevant since it can be rewritten by LS duality as an equivalent model with $\Omega$ in $] 0,1]$.
} 


\subsubsection{The Langmann-Szabo-Zarembo model}

It is a four-dimensional theory of a Bosonic complex field defined by the action

$$
S=\int \frac{1}{2} \bar{\phi}\left(-D^{\mu} D_{\mu}+\Omega^{2} x^{2}\right) \phi+\lambda \bar{\phi} \star \phi \star \bar{\phi} \star \phi
$$

where $D^{\mu}=\imath \partial_{\mu}+B_{\mu \nu} x^{\nu}$ is the covariant derivative in a magnetic field $B$.

The interaction $\bar{\phi} \star \phi \star \bar{\phi} \star \phi$ ensures that perturbation theory contains only orientable graphs. For $\Omega>0$ the $x$-space propagator still decays as in the ordinary $\phi_{4}^{4}$ case and the model has been shown renormalisable by an easy extension of the methods of the previous section [3].

However at $\Omega=0$, there is no longer any harmonic potential in addition to the covariant derivatives and the bounds are lost. Models in this category are called "critical".

\subsubsection{Critical models}

Consider the $x$-kernel of the operator

$$
\begin{aligned}
H^{-1}= & \left(p^{2}+\Omega^{2} \widetilde{x}^{2}-2 \imath B\left(x^{0} p_{1}-x^{1} p_{0}\right)\right)^{-1} \\
H^{-1}(x, y)=\frac{\widetilde{\Omega}}{8 \pi} \int_{0}^{\infty} & \frac{d t}{\sinh (2 \widetilde{\Omega} t)} \exp \left(-\frac{\widetilde{\Omega}}{2} \frac{\cosh (2 B t)}{\sinh (2 \widetilde{\Omega} t)}(x-y)^{2}\right. \\
& -\frac{\widetilde{\Omega}}{2} \frac{\cosh (2 \widetilde{\Omega} t)-\cosh (2 B t)}{\sinh (2 \widetilde{\Omega} t)}\left(x^{2}+y^{2}\right) \\
& \left.+2 \imath \widetilde{\Omega} \frac{\sinh (2 B t)}{\sinh (2 \widetilde{\Omega} t)} x \wedge y\right) \quad \text { with } \widetilde{\Omega}=\frac{2 \Omega}{\theta}
\end{aligned}
$$

The Gross-Neveu model or the critical Langmann-Szabo-Zarembo models correspond to the case $B=\widetilde{\Omega}$. In these models there is no longer any confining decay for the "long variables" but only an oscillation:

$$
Q^{-1}=H^{-1}=\frac{\widetilde{\Omega}}{8 \pi} \int_{0}^{\infty} \frac{d t}{\sinh (2 \widetilde{\Omega} t)} \exp \left(-\frac{\widetilde{\Omega}}{2} \operatorname{coth}(2 \widetilde{\Omega} t)(x-y)^{2}+2 \imath \widetilde{\Omega} x \wedge y\right)
$$

This kind of models are called critical. Their construction is more difficult, since sufficiently many oscillations must be proven independent before power counting can be established. The prototype paper which solved this problem is [21], which we briefly summarize now.

The main technical difficulty of the critical models is the absence of decreasing functions for the long $v$ variables in the propagator replaced by an oscillation, see (4.17). Note that these decreasing functions are in principle created by integration over the $u$ variables ${ }^{7}$ :

$$
\int d u e^{-\frac{\tilde{\Omega}}{2} \operatorname{coth}(2 \widetilde{\Omega} t) u^{2}+\imath u \wedge v}=K \tanh (2 \widetilde{\Omega} t) e^{-k \tanh (2 \widetilde{\Omega} t) v^{2}} .
$$

\footnotetext{
${ }^{7}$ In all the following we restrict ourselves to the dimension 2.
} 
But to perform all these Gaussian integrations for a general graph is a difficult task (see [42]) and is in fact not necessary for a BPHZ theorem. We can instead exploit the vertices and propagators oscillations to get rationnal decreasing functions in some linear combinations of the long $v$ variables. The difficulty is then to prove that all these linear combinations are independant and hence allow to integrate over all the $v$ variables. To solve this problem we need the exact expression of the total oscillation in terms of the short and long variables. This consists in a generalization of the Filk's work [8]. This has been done in [21]. Once the oscillations are proven independant, one can just use the same arguments than in the $\Phi^{4}$ case (see section 4.2) to compute an upper bound for the power counting:

Lemma 4.4 (Power counting $\mathrm{GN}_{\Theta}^{2}$ ). Let $G$ a connected orientable graph. For all $\Omega \in$ $[0,1)$, there exists $K \in \mathbb{R}_{+}$such that its amputated amplitude $A_{G}$ integrated over test functions is bounded by

$$
\begin{aligned}
& \qquad\left|A_{G}\right| \leqslant K^{n} M^{-\frac{1}{2} \omega(G)} \\
& \text { with } \omega(G)= \begin{cases}N-4 & \text { if }(N=2 \text { or } N \geqslant 6) \text { and } g=0, \\
& \text { if } N=4, g=0 \text { and } B=1, \\
N & \text { if } G \text { is critical, } \\
N+4 & \text { if } N=4, g=0, B=2 \text { and } G \text { non-critical, }\end{cases}
\end{aligned}
$$

As in the non-commutative $\Phi^{4}$ case, only the planar graphs are divergent. But the behaviour of the graphs with more than one broken face is different. Note that we already discussed such a feature in the matrix basis (see section 3.4). In the multiscale framework, the Feynamn diagrams are endowed with a scale attribution which gives each line a scale index. The only subgraphs we meet in this setting have all their internal scales higher than their external ones. Then a subgraph $G$ of scale $i$ is called critical if it has $N=4, g=0, B=2$ and that the two "external" points in the second broken face are only linked by a single line of scale $j<i$. The typical example is the graph of figure 4 a. In this case, the subgrah is logarithmically divergent whereas it is convergent in the $\Phi^{4}$ model. Let us now show roughly how it happens in the case of figure 4a but now in $x$-space.

The same arguments than in the $\Phi^{4}$ model prove that the integrations over the internal points of the graph 4 a lead to a logarithmical divergence which means that $A_{G^{i}} \simeq \mathcal{O}(1)$ in the multiscale framework. But remind that there is a remaining oscillation between a long variable of this graph and the external points in the second broken face of the form $v \wedge(x-y)$. But $v$ is of order $M^{i}$ which leads to a decreasing function implementing $x-y$ of order $M^{-i}$. If these points are true external ones, they are integrated over test functions of norm 1. Then thanks to the additional decreasing function for $x-y$ we gain a factor $M^{-2 i}$ which makes the graph convergent. But if $x$ and $y$ are linked by a single line of scale $j<i$ (as in figure $4 \mathrm{~b}$ ), instead of test functions we have a propagator between $x$ and $y$. This one behaves like (see (4.17)):

$$
C^{j}(x, y) \simeq M^{j} e^{-M^{2 j}(x-y)^{2}+\imath x \wedge y} .
$$

The integration over $x-y$ instead of giving $M^{-2 j}$ gives $M^{-2 i}$ thanks to the oscillation $v \wedge(x-y)$. Then we have gained a good factor $M^{-2(i-j)}$. But the oscillation in the 
propagator $x \wedge y$ now gives $x+y \simeq M^{2 i}$ instead of $M^{2 j}$ and the integration over $x+y$ cancels the preceeding gain. The critical component of figure 4a is logarithmically divergent.

This kind of argument can be repeated and refined for more general graphs to prove that this problem appears only when the extrernal points of the auxiliary broken faces are linked only by a single lower line [21]. This phenomenon can be seen as a mixing between scales. Indeed the power counting of a given subgraph now depends on the graphs at lower scales. This was not the case in the commutative realm. Fortunately this mixing doesn't prevent renormalisation. Note that whereas the critical subgraphs are not renormalisable by a vertex-like counterterm, they are regularised by the renormalisation of the two-point function at scale $j$. The proof of this point relies heavily on the fact that there is only one line of lower scale.

Let us conclude this section by mentionning the flows of the critical models. One very interesting feature of the non-commutative $\Phi^{4}$ model is the boundedness of its flows and even the vanishing of its beta function for a special value of its bare parameters $[22,23,24]$. Note that its commutative counterpart (the usual $\phi^{4}$ model on $\mathbb{R}^{4}$ ) is asymptotically free in the infrared and has then an unbounded flow. It turns out that the flow of the critical models are not regularized by the non-commutativity. The one-loop computation of the beta functions of the non-commutative Gross-Neveu model [43] shows that it is asymptotically free in the ultraviolet region as in the commutative case.

\subsection{Non-commutative hyperbolic polynomials}

Since the Mehler kernel is quadratic it is possible to explicitly compute the non-commutative analogues of topological or "Symanzik" polynomials.

In ordinary commutative field theory, Symanzik's polynomials are obtained after integration over internal position variables. The amplitude of an amputated graph $G$ with external momenta $p$ is, up to a normalization, in space-time dimension $D$ :

$$
A_{G}(p)=\delta\left(\sum p\right) \int_{0}^{\infty} \frac{e^{-V_{G}(p, \alpha) / U_{G}(\alpha)}}{U_{G}(\alpha)^{D / 2}} \prod_{l}\left(e^{-m^{2} \alpha_{l}} d \alpha_{l}\right) .
$$

The first and second Symanzik polynomials $U_{G}$ and $V_{G}$ are

$$
\begin{aligned}
U_{G} & =\sum_{T} \prod_{l \notin T} \alpha_{l}, \\
V_{G} & =\sum_{T_{2}} \prod_{l \notin T_{2}} \alpha_{l}\left(\sum_{i \in E\left(T_{2}\right)} p_{i}\right)^{2},
\end{aligned}
$$

where the first sum is over spanning trees $T$ of $G$ and the second sum is over two trees $T_{2}$, i.e. forests separating the graph in exactly two connected components $E\left(T_{2}\right)$ and $F\left(T_{2}\right)$; the corresponding Euclidean invariant $\left(\sum_{i \in E\left(T_{2}\right)} p_{i}\right)^{2}$ is, by momentum conservation, also equal to $\left(\sum_{i \in F\left(T_{2}\right)} p_{i}\right)^{2}$.

Since the Mehler kernel is still quadratic in position space it is possible to also integrate explicitly all positions to reduce Feynman amplitudes of e.g. non-commutative $\phi_{4}^{4}$ purely to parametric formulas, but of course the analogs of Symanzik polynomials are now hyperbolic polynomials which encode the richer information about ribbon graphs. 
The reference for these polynomials is [19], which treats the ordinary $\phi_{4}^{4}$ case. In [42], these polynomials are also computed in the more complicated case of critical models.

Defining the antisymmetric matrix $\sigma$ as

$$
\begin{aligned}
\sigma & =\left(\begin{array}{cc}
\sigma_{2} & 0 \\
0 & \sigma_{2}
\end{array}\right) \text { with } \\
\sigma_{2} & =\left(\begin{array}{cc}
0 & -i \\
i & 0
\end{array}\right)
\end{aligned}
$$

the $\delta$-functions appearing in the vertex contribution can be rewritten as an integral over some new variables $p_{V}$. We refer to these variables as to hypermomenta. Note that one associates such a hypermomenta $p_{V}$ to any vertex $V$ via the relation

$$
\begin{aligned}
\delta\left(x_{1}^{V}-x_{2}^{V}+x_{3}^{V}-x_{4}^{V}\right) & =\int \frac{d p_{V}^{\prime}}{(2 \pi)^{4}} e^{i p_{V}^{\prime}\left(x_{1}^{V}-x_{2}^{V}+x_{3}^{V}-x_{4}^{V}\right)} \\
& =\int \frac{d p_{V}}{(2 \pi)^{4}} e^{p_{V} \sigma\left(x_{1}^{V}-x_{2}^{V}+x_{3}^{V}-x_{4}^{V}\right)} .
\end{aligned}
$$

Consider a particular ribbon graph $G$. Specializing to dimension 4 and choosing a particular root vertex $\bar{V}$ of the graph, one can write the Feynman amplitude for $G$ in the condensed way

$$
\mathcal{A}_{G}=\int \prod_{\ell}\left[\frac{1-t_{\ell}^{2}}{t_{\ell}}\right]^{2} d \alpha_{\ell} \int d x d p e^{-\frac{\Omega}{2} X G X^{t}}
$$

where $t_{\ell}=\tanh \frac{\alpha_{\ell}}{2}, X$ summarizes all positions and hyermomenta and $G$ is a certain quadratic form. If we call $x_{e}$ and $p_{\bar{V}}$ the external variables we can decompose $G$ according to an internal quadratic form $Q$, an external one $M$ and a coupling part $P$ so that

$$
X=\left(\begin{array}{lllll}
x_{e} & p_{\bar{V}} & u & v & p
\end{array}\right) \quad, \quad G=\left(\begin{array}{cc}
M & P \\
P^{t} & Q
\end{array}\right),
$$

Performing the gaussian integration over all internal variables one obtains:

$$
\left.\mathcal{A}_{G}=\int\left[\frac{1-t^{2}}{t}\right]^{2} d \alpha \frac{1}{\sqrt{\operatorname{det} Q}} e^{-\frac{\widetilde{\Omega}}{2}\left(x_{e}\right.} \overline{\bar{p}}\right)\left[M-P Q^{-1} P^{t}\right]\left(\begin{array}{c}
x_{e} \\
\bar{p}
\end{array}\right) .
$$

This form allows to define the polynomials $H U_{G, \bar{v}}$ and $H V_{G, \bar{v}}$, analogs of the Symanzik polynomials $U$ and $V$ of the commutative case (see (4.22)). They are defined by

$$
\mathcal{A}_{\bar{V}}\left(\left\{x_{e}\right\}, p_{\bar{v}}\right)=K^{\prime} \int_{0}^{\infty} \prod_{l}\left[d \alpha_{l}\left(1-t_{l}^{2}\right)^{2}\right] H U_{G, \bar{v}}(t)^{-2} e^{-\frac{H V_{G, \bar{v}}\left(t, x_{e}, p_{\bar{v}}\right)}{H U_{G, \bar{v}}(t)}} .
$$

They are polynomials in the set of variables $t_{\ell}(\ell=1, \ldots, L)$, the hyperbolic tangent of the half-angle of the parameters $\alpha_{\ell}$.

Using now (4.29) and (4.30) the polynomial $H U_{G, \bar{v}}$ writes

$$
H U_{\bar{v}}=(\operatorname{det} Q)^{\frac{1}{4}} \prod_{\ell=1}^{L} t_{\ell}
$$

The main results ([19]) are 
- The polynomials $H U_{G, \bar{v}}$ and $H V_{G, \bar{v}}$ have a strong positivity property. Roughly speaking they are sums of monomials with positive integer coefficients. This positive integer property comes from the fact that each such coefficient is the square of a Pfaffian with integer entries,

- Leading terms can be identified in a given "Hepp sector", at least for orientable graphs. A Hepp sector is a complete ordering of the $t$ parameters. These leading terms which can be shown strictly positive in $H U_{G, \bar{v}}$ correspond to super-trees which are the disjoint union of a tree in the direct graph and a tree in the dual graph. Hypertrees in a graph with $n$ vertices and $F$ faces have therefore $n+F-2$ lines. (Any connected graph has hypertrees, and under reduction of the hypertree, the graph becomes a hyperrosette). Similarly one can identify "super-two-trees" $H V_{G, \bar{v}}$ which govern the leading behavior of $H V_{G, \bar{v}}$ in any Hepp sector.

From the second property, one can deduce the exact power counting of any orientable ribbon graph of the theory, just as in the matrix base.

Let us now borrow from [19] some examples of these hyperbolic polynomials. We put $s=(4 \theta \Omega)^{-1}$. For the bubble graph of figure 7 :

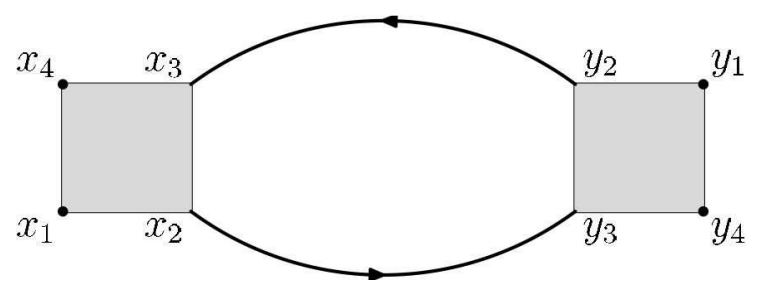

Figure 7: The bubble graph

$$
\begin{aligned}
H U_{G, v}= & \left(1+4 s^{2}\right)\left(t_{1}+t_{2}+t_{1}^{2} t_{2}+t_{1} t_{2}^{2}\right), \\
H V_{G, v}= & t_{2}^{2}\left[p_{2}+2 s\left(x_{4}-x_{1}\right)\right]^{2}+t_{1} t_{2}\left[2 p_{2}^{2}+\left(1+16 s^{4}\right)\left(x_{1}-x_{4}\right)^{2}\right], \\
& +t_{1}^{2}\left[p_{2}+2 s\left(x_{1}-x_{4}\right)\right]^{2}
\end{aligned}
$$

For the sunshine graph fig. 8:

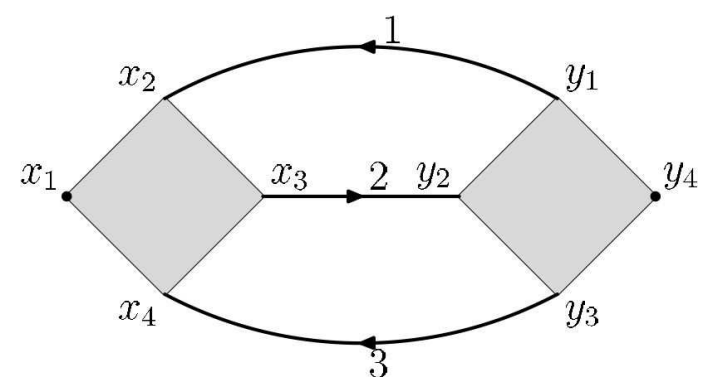

Figure 8: The Sunshine graph 


$$
\begin{aligned}
H U_{G, v}= & {\left[t_{1} t_{2}+t_{1} t_{3}+t_{2} t_{3}+t_{1}^{2} t_{2} t_{3}+t_{1} t_{2}^{2} t_{3}+t_{1} t_{2} t_{3}^{2}\right]\left(1+8 s^{2}+16 s^{4}\right) } \\
& +16 s^{2}\left(t_{2}^{2}+t_{1}^{2} t_{3}^{2}\right)
\end{aligned}
$$

For the non-planar sunshine graph (see fig. 9) we have:

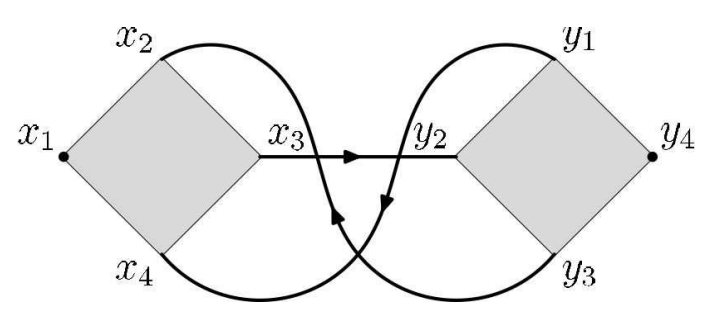

Figure 9: The non-planar sunshine graph

$$
\begin{aligned}
H U_{G, v}= & {\left[t_{1} t_{2}+t_{1} t_{3}+t_{2} t_{3}+t_{1}^{2} t_{2} t_{3}+t_{1} t_{2}^{2} t_{3}+t_{1} t_{2} t_{3}^{2}\right]\left(1+8 s^{2}+16 s^{4}\right) } \\
& +4 s^{2}\left[1+t_{1}^{2}+t_{2}^{2}+t_{1}^{2} t_{2}^{2}+t_{3}^{2}+t_{1}^{2} t_{3}^{2}+t_{2}^{2} t_{3}^{2}+t_{1}^{2} t_{2}^{2} t_{3}^{2}\right]
\end{aligned}
$$

We note the improvement in the genus with respect to its planar counterparts.

For the broken bubble graph (see fig. 10) we have:

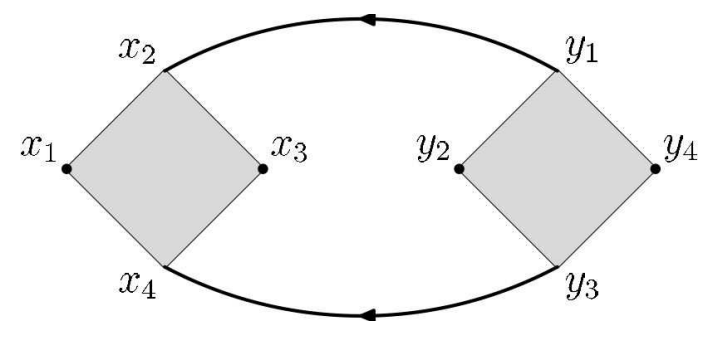

Figure 10: The broken bubble graph

$$
\begin{aligned}
H U_{G, v}= & \left(1+4 s^{2}\right)\left(t_{1}+t_{2}+t_{1}^{2} t_{2}+t_{1} t_{2}^{2}\right), \\
H V_{G, v}= & t_{2}^{2}\left[4 s^{2}\left(x_{1}+y_{2}\right)^{2}+\left(p_{2}-2 s\left(x_{3}+y_{4}\right)\right)^{2}\right]+t_{1}^{2}\left[p_{2}+2 s\left(x_{3}-y_{4}\right)\right]^{2}, \\
& +t_{1} t_{2}\left[8 s^{2} y_{2}^{2}+2\left(p_{2}-2 s y_{4}\right)^{2}+\left(x_{1}+x_{3}\right)^{2}+16 s^{4}\left(x_{1}-x_{3}\right)^{2}\right] \\
& +t_{1}^{2} t_{2}^{2} 4 s^{2}\left(x_{1}-y_{2}\right)^{2},
\end{aligned}
$$

Note that $H U_{G, v}$ is identical to the one of the bubble with only one broken face. The power counting improvement comes from the broken face and can be seen only in $H V_{G, v}$.

Finally, for the half-eye graph (see Fig. 11), we start by defining:

$$
A_{24}=t_{1} t_{3}+t_{1} t_{3} t_{2}^{2}+t_{1} t_{3} t_{4}^{2}+t_{1} t_{3} t_{2}^{2} t_{4}^{2}
$$




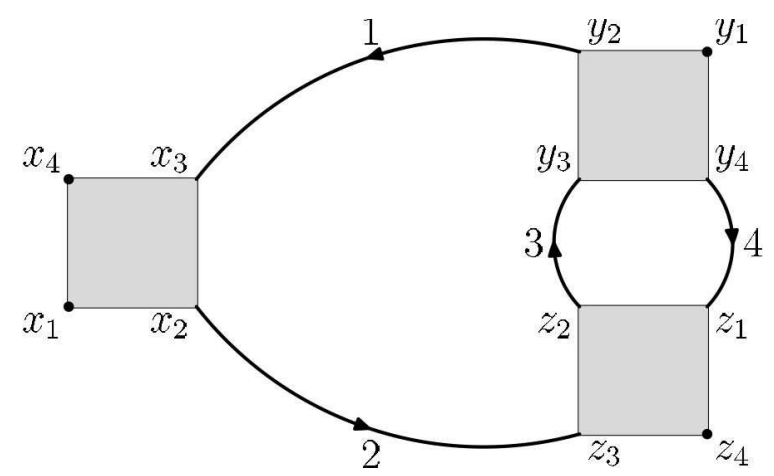

Figure 11: The half-eye graph

The $H U_{G, v}$ polynomial with fixed hypermomentum corresponding to the vertex with two external legs is:

$$
\begin{aligned}
H U_{G, v_{1}}= & \left(A_{24}+A_{14}+A_{23}+A_{13}+A_{12}\right)\left(1+8 s^{2}+16 s^{4}\right) \\
& +t_{1} t_{2} t_{3} t_{4}\left(8+16 s^{2}+256 s^{4}\right)+4 t_{1} t_{2} t_{3}^{2}+4 t_{1} t_{2} t_{4}^{2} \\
& +16 s^{2}\left(t_{3}^{2}+t_{2}^{2} t_{4}^{2}+t_{1}^{2} t_{4}^{2}+t_{1}^{2} t_{2}^{2} t_{3}^{2}\right) \\
& +64 s^{4}\left(t_{1} t_{2} t_{3}^{2}+t_{1} t_{2} t_{4}^{2}\right),
\end{aligned}
$$

whereas with another fixed hypermomentum we get:

$$
\begin{aligned}
H U_{G, v_{2}}= & \left(A_{24}+A_{14}+A_{23}+A_{13}+A_{12}\right)\left(1+8 s^{2}+16 s^{4}\right) \\
& +t_{1} t_{2} t_{3} t_{4}\left(4+32 s^{2}+64 s^{4}\right)+32 s^{2} t_{1} t_{2} t_{3}^{2}+32 s^{2} t_{1} t_{2} t_{4}^{2} \\
& +16 s^{2}\left(t_{3}^{2}+t_{1}^{2} t_{4}^{2}+t_{2}^{2} t_{4}^{2}+t_{1}^{2} t_{2}^{3} t_{3}^{2}\right) .
\end{aligned}
$$

Note that the leading terms are identical and the choice of the root perturbs only the non-leading ones. Moreover note the presence of the $t_{3}^{2}$ term. Its presence can be understood by the fact that in the sector $t_{1}, t_{2}, t_{4}>t_{3}$ the subgraph formed by the lines $1,2,4$ has two broken faces. This is the sign of a power counting improvement due to the additional broken face in that sector. To exploit it, we have just to integrate over the variables of line 3 in that sector, using the second polynomial $H V_{G^{\prime}, v}$ for the triangle subgraph $G^{\prime}$ made of lines 1,2,4.

In the critical case, it is essential to introduce arrows upon the lines and to take them into account. The corresponding analysis together with many examples are given in [42].

\subsection{Conclusion}

Non-commutative QFT seemed initially to have non-renormalisable divergencies, due to UV/IR mixing. But following the Grosse-Wulkenhaar breakthrough, there has been recent rapid progress in our understanding of renormalisable QFT on Moyal spaces. We can already propose a preliminary classification of these models into different categories, according to the behavior of their propagators:

- ordinary models at $0<\Omega<1$ such as $\phi_{4}^{4}$ (which has non-orientable graphs) or $(\bar{\phi} \phi)^{2}$ models (which has none). Their propagator, roughly $\left(p^{2}+\Omega^{2} \tilde{x}^{2}+A\right)^{-1}$ is LS covariant and has good decay both in matrix space (3.11-3.14) and direct space (4.2). 
They have non-logarithmic mass divergencies and definitely require "vulcanization" i.e. the $\Omega$ term.

- "supermodels", namely ordinary models but at $\Omega=1$ in which the propagator is LS invariant. Their propagator is even better. In the matrix base it is diagonal, e.g. of the form $G_{m, n}=(m+n+A)^{-1}$, where $A$ is a constant. The supermodels seem generically ultraviolet fixed points of the ordinary models, at which non-trivial Ward identities force the vanishing of the beta function. The flow of $\Omega$ to the $\Omega=1$ fixed point is very fast (exponentially fast in RG steps).

- "critical models" such as orientable versions of LSZ or Gross-Neveu (and presumably orientable gauge theories of various kind: Yang-Mills, Chern-Simons...). They may have only logarithmic divergencies and apparently no perturbative UV/IR mixing. However the vulcanized version still appears the most generic framework for their treatment. The propagator is then roughly $\left(p^{2}+\Omega^{2} \tilde{x}^{2}+2 \Omega \tilde{x} \wedge p\right)^{-1}$. In matrix space this propagator shows definitely a weaker decay (3.38) than for the ordinary models, because of the presence of a non-trivial saddle point. In direct space the propagator no longer decays with respect to the long variables, but only oscillates. Nevertheless the main lesson is that in matrix space the weaker decay can still be used; and in $x$ space the oscillations can never be completely killed by the vertices oscillations. Hence these models retain therefore essentially the power counting of the ordinary models, up to some nasty details concerning the four-point subgraphs with two external faces. Ultimately, thanks to a little conspiration in which the four-point subgraphs with two external faces are renormalised by the mass renormalisation, the critical models remain renormalisable. This is the main message of $[21,38]$.

- "hypercritical models" which are of the previous type but at $\Omega=1$. Their propagator in the matrix base is diagonal and depends only on one index $m$ (e.g. always the left side of the ribbon). It is of the form $G_{m, n}=(m+A)^{-1}$. In $x$ space the propagator oscillates in a way that often exactly compensates the vertices oscillations. These models have definitely worse power counting than in the ordinary case, with e.g. quadratically divergent four point-graphs (if sharp cut-offs are used). Nevertheless Ward identities can presumably still be used to show that they can still be renormalised. This probably requires a much larger conspiration to generalize the Ward identities of the supermodels.

Notice that the status of non-orientable critical theories is not yet clarified.

Parametric representation can be derived in the non-commutative case. It implies hyper-analogs of Symanzik polynomials which condense the information about the rich topological structure of a ribbon graph. Using this representation, dimensional regularization and dimensional renormalisation should extend to the non-commutative framework.

Remark that trees, which are the building blocks of the Symanzik polynomials, are also at the heart of (commutative) constructive theory, whose philosophy could be roughly summarized as "You shall use trees", but you shall not develop their loops or else you shall diverge". It is quite natural to conjecture that hypertrees, which are the natural

\footnotetext{
${ }^{8}$ These trees may be either true trees of the graphs in the Fermionic case or trees associated to cluster or Mayer expansions in the Bosonic case, but this distinction is not essential.
} 
non-commutative objects intrinsic to a ribbon graph, should play a key combinatoric role in the yet to develop non-commutative constructive field theory.

In conclusion we have barely started to scratch the world of renormalisable QFT on non-commutative spaces. The little we see through the narrow window now open is extremely tantalizing. There exists renormalizable NCQFTs eg $\phi^{4}$ on $\mathbb{R}_{\theta}^{4}$, Gross-Neveu on $\mathbb{R}_{\theta}^{2}$ and they seem to enjoy better propoerties than their commutative counterparts, for instance they no longer have Landau ghosts! Non-commutative non relativistic field theories with a chemical potential seem the right formalism for a study ab initio of condensed matter in presence of a magnetic field, and in particular of the Quantum Hall Effect. The correct scaling and RG theory of this effect presumably requires to build a very singular theory (of the hypercritical type) because of the huge degeneracy of the Landau levels. To understand this theory and the gauge theories on non-commutative spaces seem the most obvious challenges ahead of us.

\section{References}

[1] A. Connes, M. R. Douglas, and A. Schwarz, Noncommutative geometry and matrix theory: Compactification on tori, JHEP 02 (1998) 003, hep-th/9711162.

[2] N. Seiberg and E. Witten, String theory and noncommutative geometry, JHEP 09 (1999) 032, hep-th/9908142.

[3] R. Gurau, J. Magnen, V. Rivasseau and F. Vignes-Tourneret, Renormalization of non-commutative $\phi_{4}^{4}$ field theory in $x$ space, Commun. Math. Phys. 267 (2006), no. $2,515-542$, hep-th/0512271.

[4] H. Grosse and R. Wulkenhaar, Power-counting theorem for non-local matrix models and renormalisation, Commun. Math. Phys. 254 (2005), no. 1, 91-127, hep-th/0305066.

[5] H. Grosse and R. Wulkenhaar, Renormalisation of $\phi^{4}$-theory on noncommutative $\mathbb{R}^{4}$ in the matrix base, Commun. Math. Phys. 256 (2005), no. 2, 305-374, hep-th/0401128.

[6] E. Langmann and R. J. Szabo, Duality in scalar field theory on noncommutative phase spaces, Phys. Lett. B533 (2002) 168-177, hep-th/0202039.

[7] V. Rivasseau, F. Vignes-Tourneret, and R. Wulkenhaar, Renormalization of noncommutative $\phi^{4}$-theory by multi-scale analysis, Commun. Math. Phys. 262 (2006) 565-594, hep-th/0501036.

[8] T. Filk, Divergencies in a field theory on quantum space, Phys. Lett. B376 (1996) $53-58$.

[9] I. Chepelev and R. Roiban, Convergence theorem for non-commutative Feynman graphs and renormalization, JHEP 03 (2001) 001, hep-th/0008090.

[10] I. Chepelev and R. Roiban, Renormalization of quantum field theories on noncommutative $\mathbb{R}^{d}$. i: Scalars, JHEP 05 (2000) 037, hep-th/9911098. 
[11] E. Langmann, R. J. Szabo, and K. Zarembo, Exact solution of quantum field theory on noncommutative phase spaces, JHEP 01 (2004) 017, hep-th/0308043.

[12] M. R. Douglas and N. A. Nekrasov, Noncommutative field theory, Rev. Mod. Phys. 73 (2001) 977-1029, hep-th/0106048.

[13] D. Kreimer, On the hopf algebra structure of perturbative quantum field theories, Adv. Theor. Math. Phys. 2 (1998) 303-334, q-alg/9707029.

[14] A. Connes and D. Kreimer, Renormalization in quantum field theory and the riemann-hilbert problem i: The hopf algebra structure of graphs and the main theorem, Commun. Math. Phys. 210 (March, 2000) 249-273.

[15] A. Connes and D. Kreimer, Renormalization in quantum field theory and the riemann-hilbert problem ii: The $\$$-function, diffeomorphisms and the renormalization group, Commun. Math. Phys. 216 (January, 2001) 215-241.

[16] A. Connes and M. Marcolli, From physics to number theory via noncommutative geometry. ii: Renormalization, the riemann-hilbert correspondence, and motivic galois theory, hep-th/0411114.

[17] E. Leichtnam, Scaling group flow and Lefschetz trace formula for laminated spaces with \$p-\$adic transversal, ArXiv Mathematics e-prints (Mar., 2006) math/0603576.

[18] A. Abdesselam, The Jacobian conjecture as a problem of perturbative quantum field theory, Annales Henri Poincare 4 (2003) 199-215, math.co/0208173.

[19] R. Gurau and V. Rivasseau, Parametric representation of non-commutative field theory, to appear in Commun. Math. Phys, math- ph/0606030.

[20] R. Gurau, V. Rivasseau, and F. Vignes-Tourneret, Propagators for noncommutative field theories, Ann. H. Poincaré (2006) hep-th/0512071. Online first.

[21] F. Vignes-Tourneret, Renormalization of the orientable non-commutative GrossNeveu model. To appear in Ann. H. Poincaré, math-ph/0606069.

[22] H. Grosse and R. Wulkenhaar, The beta-function in duality-covariant noncommutative $\phi^{4}$-theory, Eur. Phys. J. C35 (2004) 277-282, hep-th/0402093.

[23] M. Disertori and V. Rivasseau, Two and Three Loops Beta Function of Non Commutative $\Phi_{4}^{4}$ Theory hep-th/0610224.

[24] M. Disertori, R. Gurau, J. Magnen and V. Rivasseau, Vanishing of Beta Function of Non Commutative $\Phi_{4}^{4}$ to all orders, Submitted to Phys. Lett. B, hep-th/0612251.

[25] J. M. Gracia-Bondía and J. C. Várilly, Algebras of distributions suitable for phase space quantum mechanics. I, J. Math. Phys. 29 (1988) 869-879.

[26] S. Minwalla, M. Van Raamsdonk, and N. Seiberg, Noncommutative perturbative dynamics, JHEP 02 (2000) 020, hep-th/9912072. 
[27] V. Gayral, Heat-kernel approach to UV/IR mixing on isospectral deformation manifolds, Annales Henri Poincare 6 (2005) 991-1023, hep-th/0412233.

[28] B. Simon, Functionnal integration and quantum physics, vol. 86 of Pure and applied mathematics. Academic Press, New York, 1979.

[29] H. Grosse and R. Wulkenhaar, Renormalisation of $\phi^{4}$-theory on noncommutative $\mathbb{R}^{2}$ in the matrix base, JHEP 12 (2003) 019, hep-th/0307017.

[30] H. Grosse and H. Steinacker, Renormalization of the noncommutative $\phi^{3}$ model through the kontsevich model. 2005.

[31] H. Grosse and H. Steinacker, A nontrivial solvable noncommutative $\phi^{3}$ model in 4 dimensions, hep-th/0603052.

[32] E. Langmann, R. J. Szabo, and K. Zarembo, Exact solution of noncommutative field theory in background magnetic fields, Phys. Lett. B569 (2003) 95-101, hep-th/0303082.

[33] E. Langmann, Interacting fermions on noncommutative spaces: Exactly solvable quantum field theories in 2n+1 dimensions, Nucl. Phys. B654 (2003) 404-426, hep-th/0205287.

[34] V. Rivasseau, From Perturbative to Constructive Renormalization. Princeton series in physics. Princeton Univ. Pr., Princeton, USA, 1991. 336 p.

[35] P. K. Mitter and P. H. Weisz, Asymptotic scale invariance in a massive Thirring model with $U(n)$ symmetry, Phys. Rev. D8 (1973) 4410-4429.

[36] D. J. Gross and A. Neveu, Dynamical symmetry breaking in asymptotically free field theories, Phys. Rev. D10 (1974) 3235.

[37] C. Kopper, J. Magnen, and V. Rivasseau, Mass generation in the large N Gross-Neveu model, Commun. Math. Phys. 169 (1995) 121-180.

[38] F. Vignes-Tourneret, Renormalisation des théories de champs non commutatives. Physique théorique, Université Paris 11, september, 2006.

[39] J. Polchinski, Renormalization and Effective Lagrangians, Nucl. Phys. B231 (1984) 269.

[40] M. Disertori and V.Rivasseau, Continuous constructive fermionic renormalization, Annales Henri Poincaré Vol. 1 (2000) 1 hep-th/9802145.

[41] V. Rivasseau, An introduction to renormalization, in Poincaré Seminar 2002, ed. by B. Duplantier and V. Rivasseau. Progress in Mathematical Physics 30, Birkhäuser (2003), ISBN 3-7643-0579-7.

[42] V. Rivasseau and A. Tanasa, Parametric representation of "critical" noncommutative QFT models, submitted to Commun. Math. Phys., hep-th/ 0701034. 
[43] A. Lakhoua, F. Vignes-Tourneret and J.C. Wallet, One-loop Beta Functions for the Orientable Non- commutative Gross-Neveu Model, submitted to JHEP. hep-th/0701170. 